\title{
Biomarker Discovery, Validation and Clinical Application for Patients Diagnosed with Glioma
}

\author{
Kerrie L. McDonald \\ Cure For Life Neuro-oncology Group, University of NSW \\ Australia
}

\section{Introduction}

Combined radiotherapy and chemotherapy with the alkylating agent, temozolomide plus an additional six cycles of temozolomide has been the mainstay of treatment for patients diagnosed with glioblastoma for the past 6 years. Clinically, high variability in the response to this treatment is typically observed, with some patients enjoying progression free survival for longer than others. However, tumour relapse is inevitable in the majority of patients. Local tumour recurrence, occurring within $2-3 \mathrm{~cm}$ of the original resection cavity (the area exposed to radiation treatment) is most frequently observed. Relapsed glioblastomas are typically unmanageable with median survival after recurrence of only a few months (Brandes et al. 2001). Numerous chemotherapeutic agents have been trialled in patients with recurrent glioblastomas and include enzastaurin (Wick et al. 2010), immunotherapeutic targeting of EGFRvIII (Sampson et al. 2011), cilengitide (trial ongoing) (Reardon et al. 2011), NovoTTF100A (trial ongoing), gefitinib (Uhm et al. 2010), imatinib (Dresemann et al. 2010), bevacizumab plus irinotecan (Vredenburgh et al. 2007). Only bevacizumab has shown promise for the treatment of recurrent glioblastoma, although the benefits of such a drug are still debatable. The Food and Drug Administration (FDA) in the USA approved bevacizumab for GBM under its accelerated approval process. However in Europe, the Committee for Medicinal Products for Human Use (CHMP) adopted a negative opinion.

As new therapeutic regimes are developed, it is paramount that we develop a strategy for identifying the patients that will show a positive response to treatment. The recognition and validation of biomarkers of clinical response is important for several reasons: to avoid unnecessary toxicity in patients that fail to respond to the particular treatment; to reduce the colossal cost to healthcare which is typically associated with targeted therapy and most importantly, to better understand drug resistance. This improved knowledge could lead to new strategies to overcome the initial resistance and identify synergistic drug combinations.

\subsection{Prognostic and predictive biomarkers}

Hopes for progressing curative treatment programs for cancer patients centre on the development and successful implementation of personalised medicine. Personalised medicine hinges on biomarkers which are highly sensitive and highly specific in revealling information that is relevant for diagnosis, prognosis and therapy. The most sought after biomarkers are the ones that can identify which patients are at high risk of tumour relapse 
and developing cytotoxicity to specific chemotherapeutic agents. The use of biomarkers to identify patients who don't respond to treatment early could confer enormous benefits for patients diagnosed with glioblastoma, especially considering the short survival time. Many biomarkers have shown excellent utility in survival prognostication but not necessarily at the level of influencing an oncologist's decision to administer a specific drug or alter the treatment schedule (Figure 1). In addition, another challenge in oncology is the translation of prospective biomarkers from the lab into validated diagnostic tests.

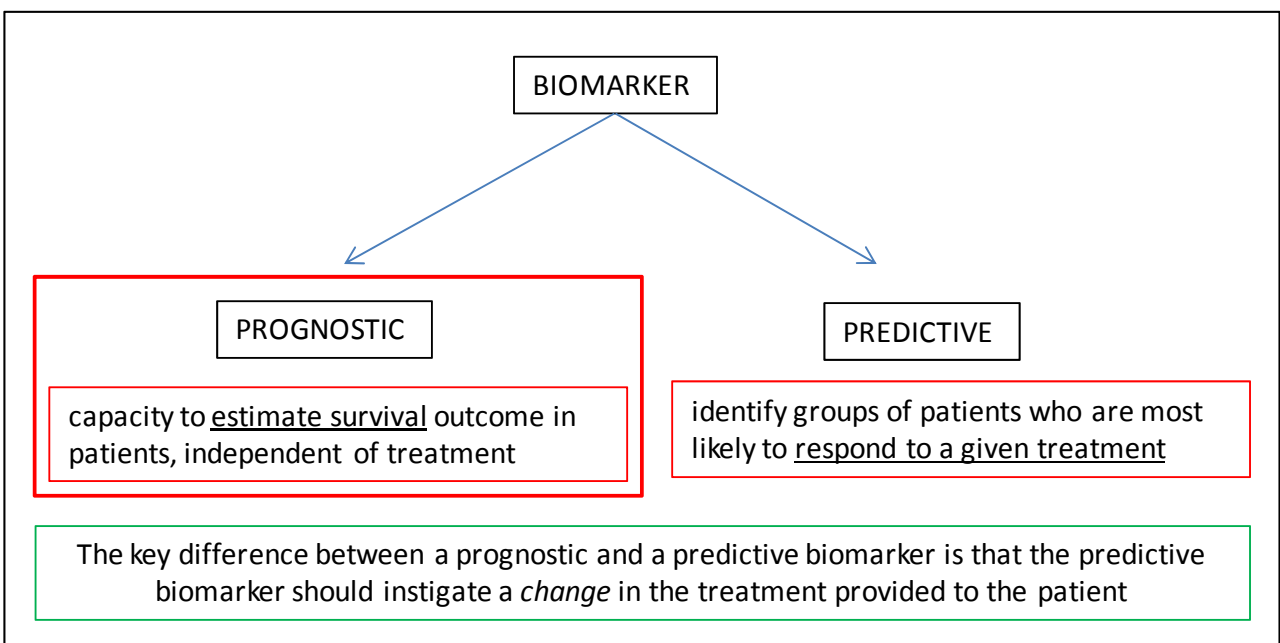

Fig. 1. Schematic overview of the key difference between biomarkers with prognostic and predictive qualities. Prognostic markers are more common in glioblastoma.

Most biomarkers often have both prognostic and predictive value. There is no strict rule when it comes to what constitutes a biomarker. A marker can consist of genomic and proteomic patterns, single genes or proteins, chromosomal abnormalities, epigenetic signatures, aberrant microRNA as well as imaging changes observed on a MRI or PET scan. A prognostic marker has the capacity to estimate survival outcome in patients, independent of treatment. The genetic profiling of large tumour cohorts with comprehensive clinical and survival data have promoted the discovery of novel molecular biomarkers associated with survival, in addition to traditional clinical and morphological features. Examples of biomarkers with prognostic significance include amplification of Epithelial Growth Factor Receptor (EGFR) (Shinojima et al. 2003; Layfield et al. 2006; Kaloshi et al. 2007; Gan et al. 2009; Inda et al. 2010), over-expression of chitinase-3-like-1 (CH3L1 or YKL-40) (Hormigo et al. 2006; Pelloski et al. 2007), osteopontin (Sreekanthreddy et al. 2010), loss of phosphatase and tensin homolog (PTEN) (Hill et al. 2003; Parsa et al. 2007) and mutations in the tumour suppressor protein, p53 (Shiraishi et al. 2002; Ruano et al. 2009). Prognostic biomarkers have great utility in the clinic. Not only do these markers present as potential therapeutic targets but they can be used to pool groups of glioma with similar genetic profile. This enrichment of the test population leads to increased homogeneity and a much more uniform response to treatment (Figure 2). 


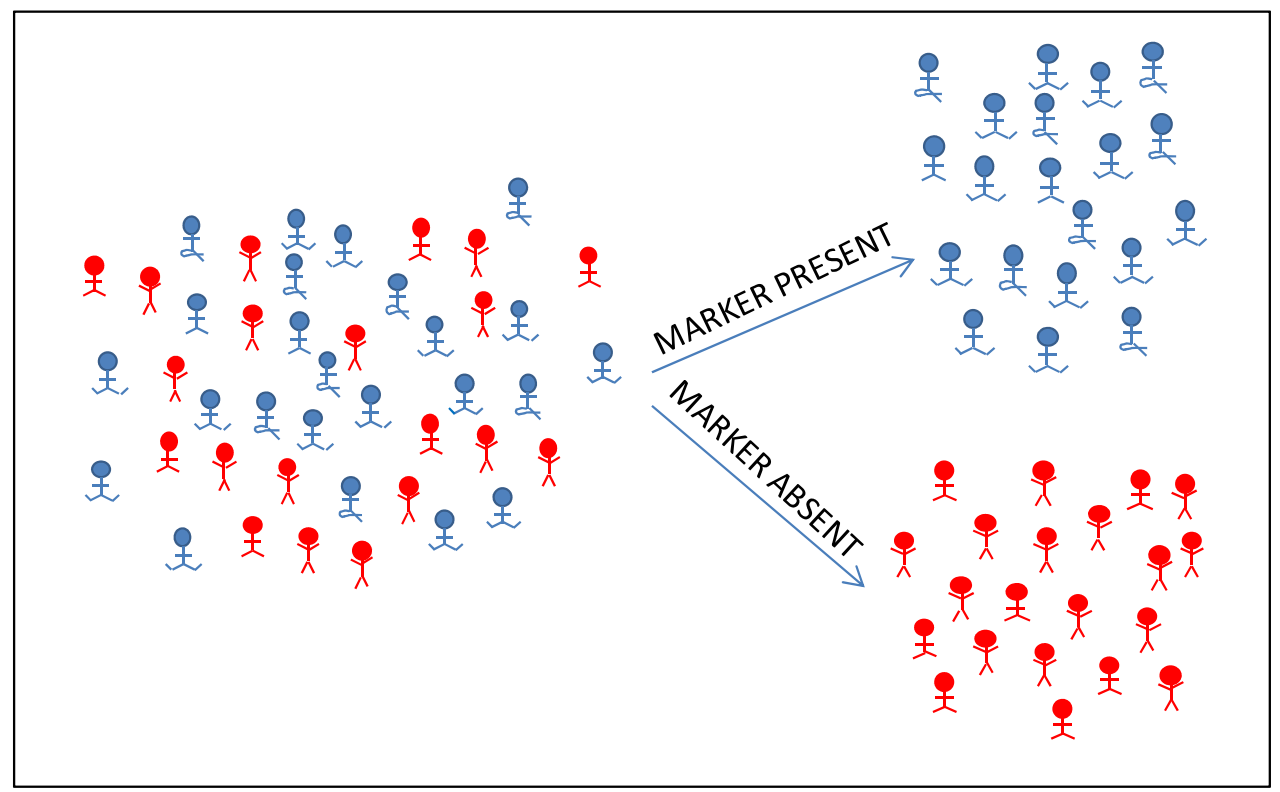

Fig. 2. Molecular diagnostics allows for the identification of GBM subgroups with similar genetic profile. This enrichment allows for a more uniform tumour response.

Much more difficult to identify are biomarkers with predictive power in the context of a specific therapy. Predictive biomarkers are markers which can be used to identify groups of patients who are most likely to respond to a given treatment. The key difference between a prognostic and a predictive biomarker is that the predictive biomarker should instigate a change in the treatment provided to the patient (Figure 1). Estrogen Receptor (ER) status in patients with breast cancer strongly predicts treatment response to tamoxifen (Kurokawa et al. 2000; Hu\&Mokbel 2001). Additionally, patients with variant forms of the gene CYP2D6 (also called simply 2D6) may not receive full benefit from tamoxifen because of the slow metabolism of the tamoxifen prodrug into its active metabolite 4-hydroxytamoxifen (Goetz 2010; Stingl et al. 2010; de Souza\&Olopade 2011). Approximately 60\% of malignant melanomas harbour the BRAF mutation. Although patients with the damaged BRAF are non-responsive to the KRAS/BRAF inhibitor, sorafenib, response to the second-generation drug called PLX4720 is favourable (Whittaker et al. 2010). Improved outcomes have also been reported in patients with non-small cell lung cancer (NSCLC) harbouring EGFR mutations treated with the tyrosine kinase inhibitors (TKI) erlotinib and gefitinib (Kim et al. 2008; Paz-Ares et al. 2010).

In a highly heterogeneous tumour such as glioblastoma, the search for predictive markers to treatment for use in clinical trials and in every day clinic has been disappointing.

\subsection{Molecular subtypes of glioblastoma}

Most centres around the world use the World Health Organisation (WHO) grading of tumours of the central nervous system (Fuller\&Scheithauer 2007). Glioma grade is defined by the presence or absence of histopathological features, namely: nuclear pleomorphism, mitoses, proliferative index and necrosis and/or microvascular proliferation. A significant 
limitation to this histopathology-based analysis is its inability to detect functional differences occurring on the subcellular level. This is evidenced by the high variability observed in the clinical outcomes in patients with the same diagnosis and differences in response to therapy. To advance survival times and clinical treatment of these patients with an, on average, dismal prognosis molecular markers with capacity to take into consideration the high molecular heterogeneity are needed in the clinic.

The wide spectrum of molecular difference in glioblastoma is evident from global expression studies, in particular, the molecular cataloguing project: The Cancer Genome Atlas (TCGA) (2008). Surveying the mutational environment of glioblastoma revealed that aberrations occur most commonly in genes whose protein products regulate the core cell growth signalling pathways that are already known to be important such as EGFR, PTEN, p53 and CDKN2A. What this survey did reveal was the extent of genomic complexity. Each tumour harbours different mutations. In addition, we are beginning to appreciate that the core pathways of cancer are not linear, rather complex and interacting. Given this complexity, it is very unlikely that a single genetic change will predict treatment response.

Gene expression profiling has provided an opportunity to further define prognostic and predictive factors (Settle\&Sulman 2011). Gene signatures have successfully categorised glioblastomas that histologically appear indistinguishable, into molecular subgroups which often have very different clinical outcomes (Colman et al. 2010; Verhaak et al. 2010). Based on survival associated genes, 76 high grade gliomas were classified into the broad genotology groups; proneural, mesenchymal and proliferative (Phillips et al. 2006). The use of larger and multiple datasets have refined these subtypes into two broad groups, proneural and mesenchymal angiogenic (Colman et al. 2010). Overexpression of a mesenchymal gene expression signature and loss of a proneural signature are associated with a poor prognosis group. By subtyping glioblastoma into mesenchymal and proneural subtypes, the sameness of patient populations is improved. In addition, the genes belonging to each group provides biologists hints for therapeutic targeting. For example, the mesenchymal subtype of glioblastoma is over-represented by genes involved in angiogenesis and invasion (Colman et al. 2010). This subgroup of patients is more responsive to bevacizumab. Mutation in the isocitrate dehydrogenase 1 (IDH1) gene is strongly associated with the proneural subtype of glioblastoma and a much better prognosis (Noushmehr et al. 2010). Increasing evidence suggests that proneural glioblastomas have a different histogenic origin which is further supported by the recent discovery of a gliomaCpG island methylator phenotype (G-CIMP) (Noushmehr et al. 2010). Both IDH1mt and the G-CIMP have a higher incidence in secondary GBMs which arise from a prior, lower grade lesion. MGMT promoter methylation, G-CIMP and mutations in IDH1 are all prognostic. Although a correlation between proneural GBM subtypes and specific treatment has not been determined, it has been suggested by a few studies that chemotherapy agents such as temozolomide and others targeted at cell growth may not be as effective for this group as previously thought (Verhaak\&Valk 2010).

\section{Prognostic biomarkers in glioma}

Molecular markers identified to hold prognostic significance in glioma include loss of heterozygosity of the chromosomal arms $1 p$ and $19 q(\mathrm{LOH} 1 \mathrm{p} / 19 \mathrm{q})$, methylguanine methyltransferase (MGMT) promoter methylation, mutations in the isocitrate dehydrogenase 1 (IDH1) gene, mutations in TP53, loss of PTEN activity, amplification of 
EGFR, presence of the EGFR delta variant (EGFRvIII) and overexpression of chitinase 3-like 1 (YKL40). Gene profiling and cross validation in multiple independent datasets has resulted in the separation of glioblastoma into two major subgroupings: proneural and mesenchymal. The proneural tumours have a much better survival outlook and can be further characterised by the presence of a glioma $\mathrm{CpG}$ island methylation phenotype (gCIMP).

Extensive reviews of EGFR, PTEN and TP53 are covered elsewhere. This discussion will focus on LOH 1p/19q, MGMT promoter methylation and mutations in IDH.

\subsection{Loss of heterozygosity $1 p$ and $19 q$}

A hallmark of oligodendroglial tumours is the co-deletion of the chromosomal arms $1 \mathrm{p}$ and $19 q$ corresponding to an unbalanced translocation $t(1 ; 19)$ ( $q 10 ; p 10)$. This can be readily detected using Fluorescence In situ hybridisation (FISH) (Figure 3). $\mathrm{LOH}$ at $1 \mathrm{p} 19 \mathrm{q}$ is observed in up to $69 \%$ of grade II and grade III (anaplastic) oligodendrogliomas and is far more common in 'pure' oligodendroglioma than astrocytoma and mixed oligoastrocytoma (Barbashina et al. 2005). LOH of $1 \mathrm{p} 19 \mathrm{q}$ confers a clear survival advantage in anaplastic oligodendroglioma and mixed oligoastrocytoma however the survival advantage conferred for grade II lesions is less clear (Laigle-Donadey et al. 2005; Jenkins et al. 2006; Walker et al. 2006). Whether the co-deletion mediates a prognostic advantage or results in a heightened sensitivity to radiation and chemotherapy is unknown. In general, oligodendrogliomas with $\mathrm{LOH}$ at $1 \mathrm{p} 19 \mathrm{q}$ represent a group of highly chemosensitive gliomas, especially to the combination of procarbazine, lomustine (CCNU), and vincristine (PCV).

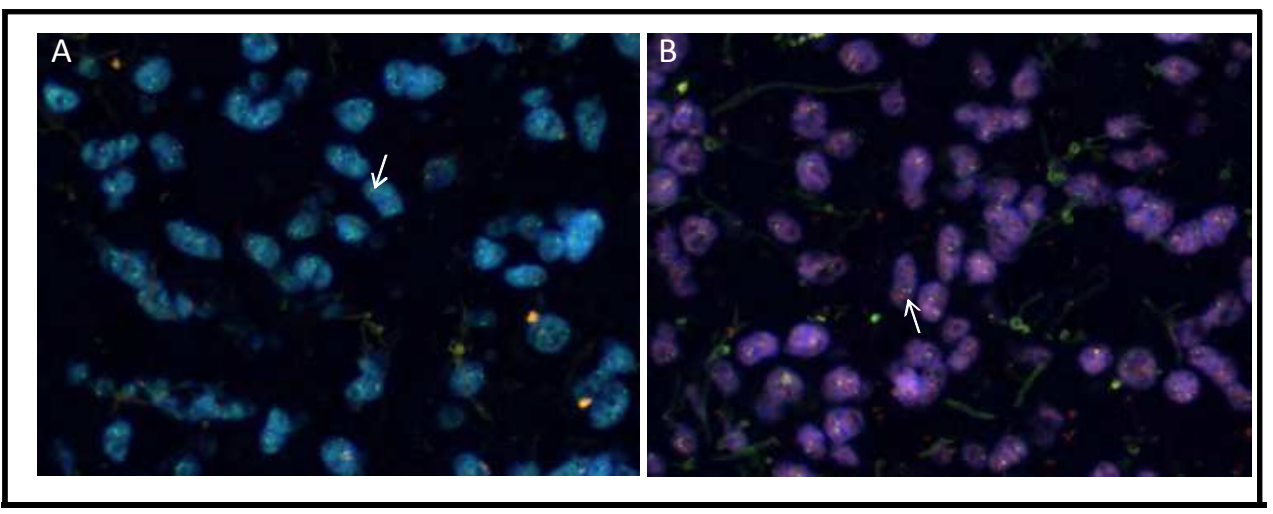

Fig. 3. Representative photomicrographs of loss of $1 p(A)$ and loss of $19 q$ (B) chromosomal arms detected using FISH. Arrow indicates only one chromosome copy instead of the expected two. Photomicrographs were kindly donated by Dr Michael Buckland, Department of Neuropathology, University of Sydney.

The standard treatment for anaplastic oligodendrogliomas consists of complete surgical removal where possible followed by radiation therapy and chemotherapy, typically with temozolomide because it is well tolerated. It is generally accepted that chemotherapy is of value in the treatment of patients with anaplastic oligodendrogliomas (Mokhtari et al. 2011). 
Because of the potential toxicity to the CNS, many clinicians have suggested that radiotherapy treatment may be better reserved for progressive disease. Treatment with temozolomide is now favoured over PVC treatment because of its low toxicity. Studies treating anaplastic oligodendroglioma patients with temozolomide have also found that the presence of $\mathrm{LOH}$ at $1 \mathrm{p} / 19 \mathrm{q}$ is a favourable predictive marker (Brandes et al. 2006; Mikkelsen et al. 2009; Ramirez et al. 2010). This could also be because the majority of oligodendrogliomas harbouring $\mathrm{LOH}$ at $1 \mathrm{p} / 19 \mathrm{q}$ also show methylation in the promoter region of MGMT. Clinical studies have been designed to establish whether combining or adding chemotherapy to radiotherapy is of benefit to oligodendroglioma patients or whether these patients could benefit from upfront chemotherapy (without radiotherapy).

Two large prospective trials have shown little benefit for adding adjuvant PVC before radiotherapy (Cairncross et al. 2006) or after radiotherapy (van den Bent et al. 2006). To address whether treatment of oligodendrogliomas with chemotherapy alone is feasible and safe, the NOA-04 Phase III trial compared radiotherapy versus chemotherapy with either PCV or temozolomide as initial therapy in 318 patients with anaplastic gliomas (WHO grade 3) (randomly assigned 2:1:1 to receive radiotherapy (arm A) or chemotherapy with either PCV (arm B1) or temozolomide (arm B2)) (Wick et al. 2009). The clinical relevance of 1p/19q codeletion, ${ }^{6}$-methylguanine DNA-methyltransferase (MGMT) promoter methylation, and IDH1 mutations in codon 132 in these tumours were also measured and analysed. This important trial confirmed that there was no survival difference in administering initial radiotherapy or initial chemotherapy (Wick et al. 2009). One very important finding to emerge from the study was the presence of mutations in IDH1 provided the best prognostic model. An ongoing EORCT 26081 Phase III trial of radiotherapy, temozolomide and concomitant and adjuvant temozolomide in patients with anaplastic oligodendrogliomas with $1 p / 19 q$ codeletions will further confirm what the optimal treatment for these tumours is (more information below).

The gene products that are affected as a result of $\mathrm{LOH}$ remain under investigation and may include mediators of cytotoxic resistance or may represent an early oncogenic lesion still retaining sensitivity to genotoxic agents or insults. Microarray technology has been used to profile gene expression in oligodendrogliomas to look for putative tumour suppressor gene candidates and genes which could mediate the observed chemosensitivity using a variety of microarray platforms (Mukasa et al. 2002; Nutt et al. 2003; Mukasa et al. 2004; Tews et al. 2006; Tews et al. 2007; Ducray et al. 2008). These studies have identified some interesting gene candidates located on the $1 \mathrm{p}$ and $19 \mathrm{q}$ chromosomal arms however none have gone on to be validated prospectively. Interestingly, these profiling experiments identified a proneural signature associated with $1 \mathrm{p} 19 \mathrm{q}$ codeleted oligodendrogliomas and a better survival outcome (Phillips et al. 2006). In contrast, the mesenchymal signature is more commonly associated with glioblastoma (discussed in more detail below). Noteworthy is the absence of EGFR amplifications in the proneural group. Ducray and colleagues compared $1 \mathrm{p} 19 \mathrm{q}$ codeleted gliomas to EGFR-amplified gliomas and found that the proneural gene internexin (INA) which encodes neurofilament-interacting protein was significantly differentially expressed (Ducray et al. 2009). The prognostic significance of INA was further assessed and confirmed in the prospective, randomized EORTC 26951 trial of adjuvant PVC (Mokhtari et al. 2011). INA strongly correlated with 1p19q codeletion, mutated IDH1 and MGMT promoter methylation. 


\subsection{MGMT}

The O6-methylguanine-DNA methyltransferase gene, MGMT, located on chromosome 10q26.1 encodes a DNA repair protein that restores mutagenic $\mathrm{O}^{6}$-alkylguanine to normal guanine within genomic DNA. $\mathrm{O}^{6}$-alkylguanines can pair erroneously with thymine during DNA replication, resulting in G:C>A:T transitions, as well as causing cross-links between adjacent strands of DNA, both of which can lead to neoplastic transformation (Gerson 2004). MGMT thus protects cells from the toxic and carcinogenic effects of alkylating agents and is absent in many types of human malignancy. Loss of MGMT protein expression is frequently associated with transcriptional silencing of the MGMT gene by methylation of its CpG island promoter in various neoplasia, (Esteller et al. 1999) as exemplified by $35-55 \%$ of gliomas (Silber et al. 1998; Esteller et al. 2000; Nakamura et al. 2001; Kamiryo et al. 2004; Paz et al. 2004; Brell et al. 2005; Hegi et al. 2005). However, several large studies of glioma have shown the correlation between immunohistochemical loss of MGMT and promoter methylation is not always correlative (Preusser et al. 2008; Cao et al. 2009; Hawkins et al. 2009; Mellai et al. 2009).

Alkylating drugs such as temozolomide are used in chemotherapy for the targeted cell death of rapidly-replicating neoplastic cells and MGMT expression is a key factor in conferring resistance to these agents. In 2005, a new treatment regime was developed and tested in a randomised, phase III clinical trial whereby the alkylating agent, temozolomide was combined with radiotherapy (RT) in concurrent treatment followed by an additional 6 cycles of Temozolomide for newly diagnosed glioblastoma (Stupp et al. 2005). This was the first trial to achieve a clinically meaningful and statistically significant overall median survival benefit of 2.5 months when compared to radiotherapy alone. More compelling were the two-year survival rates with $26 \%$ of patients treated with concurrent treatment still alive after two years compared with just $10.4 \%$ for patients treated with radiotherapy alone. These survival benefits were still apparent after 5 years of follow-up (Stupp et al. 2009).

The molecular basis for the differential response of glioblastoma patients to temozolomide has been recognized. Temozolomide is an oral alkylating chemotherapy which is spontaneously converted into its active metabolite and readily crosses the blood-brain barrier. The primary mode of action of temozolomide is to damage the DNA by introducing alkyl adducts. These cause genetic mutations as well as cross-links between DNA strands that inhibit DNA replication and thereby trigger cell death. Thus alkylating agents target rapidly replicating neoplastic cells. However, while temozolomide introduces alkyl adducts into DNA, MGMT reverses them. Thus tumour cells expressing MGMT are chemoresistant to this class of drugs (Pegg 1990). In a companion laboratory study to the phase III trial combining radiotherapy with temozolomide, Hegi et al. demonstrated a pronounced positive survival response in patients whose tumours had lost MGMT by promoter methylation. Strikingly, patients whose tumours were MGMT-methylated demonstrated extended overall and progression-free survival compared to those whose tumours were unmethylated, and therefore MGMT methylation was postulated to be a positive predictor of patient response to alkylating agents (Esteller et al. 2000; Hegi et al. 2005). Since these seminal reports in 2005, the standard of care for patients diagnosed with glioblastoma has comprised surgery with maximal feasible resection and radiotherapy with concurrent and adjuvant temozolomide. Yet widespread adoption of MGMT methylation as a marker of response to temozolomide in clinical practice has not transpired. 


\subsubsection{Routine testing for MGMT methylation}

While MGMT methylation could be routinely used as a prognostic/predictive marker in glioblastoma, there is so far no consensus on the method to be applied. Assessment of MGMT promoter methylation is difficult due to the complex nature of the techniques involved. To detect methylation, bisulfite treatment of the DNA is required, a process that may result in degradation of DNA and subsequent low success rates in PCR. This is further compounded by the fact that the most commonly available tissue for assessment is formalin fixed paraffin embedded (FFPE), and the DNA subsequently extracted from this tissue is usually fragmented, again making PCR more difficult.

Promoter methylation analysis by qualitative methyl-specific polymerase chain reaction (MSP) or semi-quantitative methyl-specific polymerase chain reaction (SQ-MSP), especially from FPPE tissue is technically demanding. MSP is the more limited because the methylation status of only a few CpG sites (i.e., those interfering with the PCR primer binding) can be interrogated at once. The technique also has the drawback of providing only a qualitative indication of the methylation status of the sites. Karayan-Tapon (KarayanTapon et al. 2010) evaluated MGMT promoter methylation using MSP, SQ-MSP and pyrosequencing. The best predictive value for overall survival was obtained by pyrosequencing. Pyrosequencing technology is a technique that generates a quantitative measure of methylation and automatically calculates and reports percent methylation for each CpG site in the studied sequence, thus allowing detection of partially methylated CpG sites.

There are other methodologies for assessing the promoter methylation of MGMT. The testing needs to be resolved for MGMT to be used routinely in the clinic and perhaps a surrogate marker of MGMT such as another protein product readily visualised by immunohistochemistry or a polymorphism detected in blood may be the way forward.

\subsubsection{Strategies to overcome MGMT activity}

With the recognition that an unmethylated MGMT promoter is associated with a poorer response to temozolomide, strategies have evolved to circumvent the resistance that MGMT confers. Combination therapy with multiple chemotherapeutic drugs known to deplete MGMT (specifically procarbazine and temozolomide) has been successfully assessed in a Phase I trial (Newlands et al. 2003) but as yet has not been shown to confer a benefit in survival. $\mathrm{O}^{6}$ benzylguanine ( $\left.\mathrm{O}^{6} \mathrm{BG}\right)$, a substrate for MGMT, has also been used to decrease MGMT levels. However, systemic administration of $\mathrm{O}^{6} \mathrm{BG}$ has been associated with significant toxicity, thereby necessitating a reduction in chemotherapy dose (Quinn et al. 2002; Quinn et al. 2005). A recent case report of local administration of $\mathrm{O}^{6} \mathrm{BG}$, allowing the systemic effects to be avoided, shows some promise (Koch et al. 2007).

Alteration of temozolomide dosing regimens from the usual method of 5 days of treatment every 28 days to more frequent, lower-dose treatment has been evaluated. Protracted temozolomide exposure may reduce MGMT activity. Brock and colleagues demonstrated safety of a low dose of temozolomide for up to 49 consecutive days, however the efficacy of this lower dose is unclear (Brock et al. 1998). Depletion of peripheral mononuclear MGMT has been demonstrated with more prolonged dosing regimens and unfortunately this has been associated with profound lymphocytopaenia and opportunistic infections (Tolcher et al. 2003; Wick et al. 2004; Wick\&Weller 2005). More recent evidence suggest that daily dosing may be associated with improved outcome (Buttolo et al. 2006). Additionally, a dosing regimen of 14 days of treatment out of every 28 days has not only been shown to 
lead to a progression free survival benefit, outcome with this treatment regimen was not significantly associated with MGMT promoter methylation (Wick et al. 2007).

Treating patients with continuous $50 \mathrm{mg} / \mathrm{m}^{2}$ at relapse after a standard temozolomide schedule of $150-200 \mathrm{mg} / \mathrm{m}^{2}$ resulted in a PFS6 of $47-57 \%$ (Perry et al. 2008). The efficacy and safety of this continuous dose-intense temozolomide schedule for recurrent GBM was tested in a multicenter, phase II study, RESCUE. Overall, PFS6 in 116 patients with recurrent GBM was $24 \%$ (Perry et al. 2010). Not surprisingly, the best responding patients were those who were treated with conventional chemoradiotherapy. However, what was interesting was the similar benefit to treatment recorded in the patients who experienced early progression on standard therapy (Perry et al. 2010).

\subsection{IDH mutations}

The Cancer Genome Atlas (TCGA) efforts made the initial breakthrough discovery that $11 \%$ of glioblastomas harbour point mutations in cytoplasmic and mitochondrial NADP+dependent isocitrate dehydrogenases 1 and 2 (IDH1 and IDH2) (Balss et al. 2008; Parsons et al. 2008; Dang et al. 2009). The normal function of the IDH enzymes is to convert isocitrate into a-ketoglutarate. Mutations, specifically at the arginine 132 (R132) codon, are more frequently observed in low grade and anaplastic gliomas and secondary glioblastomas (5093\%) than mutations found in IDH2 [arginine 172 (R172) codon] (3-5\%). No gliomas have been found to have point mutations in both IDH1 and IDH2 (Yan et al. 2009).

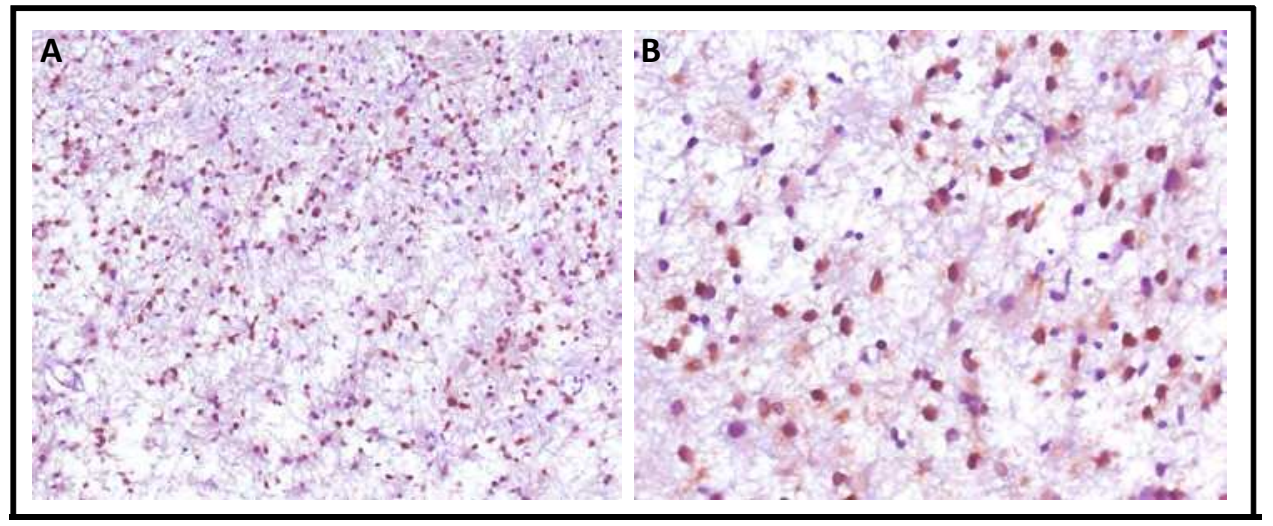

Fig. 4. Representative photomicrographs of IDH1 mutations detected with the Anti-Human IDH1 R132H Mouse Monoclonal Antibody (DIA-H09M) at x20 magnification (A) and x40 (B) Photomicrographs were kindly donated by Dr Michael Buckland, Department of Neuropathology, University of Sydney.

Hartmann and colleagues used an antibody which specifically detected the R132 mutation in IDH1 allowing assessment with simple immunohistochemistry (Hartmann et al. 2010). (Figure 4). The mutation was detected in $72 \%$ low grade astrocytomas (AII; $n=227$ ); $64 \%$ anaplastic astrocytomas (AA; $n=228$ ); 82\% low grade oligodendroglioma (OII; $n=128) ; 70 \%$ anaplastic oligodendroglioma ( $\mathrm{AO} ; \mathrm{n}=174) ; 82 \%$ low grade oligoastrocytomas (OAII; $\mathrm{n}=76$ ); $66 \%$ anaplastic oligoastrocytoma (AOA; $n=177$ ) and $9 \%$ glioblastoma (GBM; $=521$ ). What was most significant about this study was the progression free and overall survival curves. 
In order of most favourable to poor survival: (1) AA with IDH1 mutation, (2) GBM with IDH1 mutation, (3) AA with IDH1 wild type and (4) GBM with IDH1 wild type. Routine testing for IDH1 mutations will have clinical ramifications regarding histological diagnosis and treatment schemes. The IDH1 mutation is of greater prognostic relevance than histopathological diagnosis according to the World Health Organisation (WHO) classification system (Hartmann et al. 2010). Subsidised treatment schemes approved for glioblastoma such as concomitant radiotherapy and temozolomide and bevacizumab (USA only) may need to be revised to allow anaplastic gliomas with IDH1 wild type status to be treated.

Mutations of the codons in IDH1 and 2 lead to a loss in the production of a-ketoglutarate and a gain of the catalytic activity to produce 2-hydroxyglutarate (2-HG) (Xu et al. 2011). 2HG levels are highly elevated in IDH-mutated cancers and lead to genome wide histone and DNA methylation alterations (Xu et al., 2011; Dang et al., 2009). Hypermethylation at a large number of loci have been associated with IDH-mutated glioma suggesting that IDH mutation is associated with a distinct DNA methylation phenotype (Noushmehr et al. 2010; Christensen et al. 2011). GoldenGate array methylation data was obtained from 131 glioma patients (all types and histological grades) to interrogate methylation patterns associated with IDH mutation and survival. IDH1 mutations were present in $60 \%$ of tumours. Distinct differences between the numbers of significantly differentially hypermethylated loci were noted in IDH mutant tumours compared to IDH wild type tumours. Specific to IDH mutant tumours, cellular signalling pathways were hypermethylated whilst metabolism and biosynthesis pathways were hypermethylated (Christensen et al. 2011). This might be compensatory for the metabolic stress related to the mutation.

In a series of elegant in vitro-based experiments, Yan's group transformed human oligodendroglial (HOG) cells with IDH1-R132 or treated cells with 2-HG (Yan et al. 2009). They noted changes in gene expression common to both IDH1-R132 cells and 2-HG-treated cells when compared to IDH1-wildtype and untreated cells, implying that these changes were the result of increased 2HG (Reitman et al. 2010; Reitman\&Yan 2010). However, reductions in glutamate and several glutamate-related metabolites were observed exclusively in the IDH1-R132 cells. Particular attention was paid to reduced levels of a common dipeptide in the brain, N-acetyl-aspartyl-glutamate (NAAG), however its contribution to pathogenesis remains unclear (Reitman et al. 2011).

Recently, IDH mutations have been shown to be tightly associated with the presence of a glioma CpG island methylator phenotype (gCIMP) (Noushmehr et al. 2010). CIMPs are characterised by highly concordant DNA methylation of a subset of loci. Improved survival was observed in gliomas with IDH1 mutation and positive for gCIMP suggesting that there are molecular features within gCIMP gliomas that encourage a less aggressive phenotype. CIMP positive colon cancers also have a better prognosis. It is not known whether glioma cells acquire the mutation in IDH1 which then leads to genome histone and DNA methylation patterns, reflected by the presence of a gCIMP or that transcriptional silencing of gCIMP targets may provide the optimal environment for gliomas to acquire the mutation (genomic instability) (Noushmehr et al. 2010).

Gliomas with IDH1 mutations as well as the presence of gCIMP displayed significantly better overall survival (median survival: 2.9 years) compared to all other patients (median survival: 1.04 years). The favourable survival observed in IDH1 mutation-gCIMP positive gliomas may be because these tumours are highly represented in the proneural subset of gliomas. Clinically, the prognostic utility of IDH1 mutations emerged in the NOA-04 trial. 
IDH1 mutations conferred a significantly longer time to treatment failure (TTF) which was independent of histology, treatment, codeletion of $1 p$ and $19 q$ and MGMT promoter methylation status (Wick et al. 2009). IDH1 mutations as well as the gCIMP represent a significant breakthrough in how we diagnose patients. Testing for IDH1 mutations has quickly translated into routine diagnostic use. No doubt, IDH1 mutations and perhaps the gCIMP will be used to stratify patients for future clinical trials. Attention has shifted to examining therapeutic targets for IDH1 as well as the possibility of inducing mutations in IDH1 in GBMs that don't possess the mutation.

\section{Use as predictive biomarkers}

Biomarkers which can foretell whether patients are resistant to a certain treatment and predict drug sensitivity are urgently needed. The success rate of matching biomarkers with treatments has been less than satisfactory. Fewer than 100 biomarkers have been validated for routine clinical practice, despite the publication of more than 150,000 claimed biomarkers. Impeding the successful translation of biomarkers into the clinical setting is non-standardised biological specimen and clinical data collection, particularly clinical information pertaining to drug sensitivity and progression free survival. In addition, far greater numbers of tumour specimens from patients treated uniformly may be needed to be analysed than what we previously assumed.

The only biomarker with reported predictive value is MGMT promoter methylation. As discussed earlier in the MGMT section, the role of MGMT is to protect cells from alkylating damage specifically by removing the alkyl adducts from the $\mathrm{O}^{6}$ position of guanine and the $\mathrm{O}^{4}$ position of thymine and effectively restoring the DNA bases and prevent TMZ-induced cell death. However, the present NOA-04 trial does not support the suggestion that MGMT promoter methylation is simply predictive for response to alkylating chemotherapy (Wick et al. 2009). NOA-04 showed a striking difference in PFS between patients with versus without MGMT promoter methylation who were treated with radiotherapy alone. Thus MGMT promoter hypermethylation in anaplastic gliomas may be regarded as (1) a prognostic marker for good outcome in patients treated with radiotherapy or (2) predictive for response to radiotherapy itself.

The most significant issue with implementing MGMT promoter methylation as a predictive test for TMZ therapy is that there is currently no alternative treatment strategy available for those patients with unmethylated MGMT tumours. Until alternative treatments are available and the MGMT test is more reliable and robust, will clinicians consider MGMT promoter methylation as a predictive test.

\section{Use of biomarkers in clinical trials}

Co-deletion of the chromosomal arms $1 p$ and $19 q$ is a requirement for entry of anaplastic gliomas into the CODEL study which is assessing the role of concomitant and adjuvant temozolomide added to standard radiotherapy and has temozolomide monotherapy in an observation arm. A phase III randomized sister study to CODEL, CATNON, examines radiotherapy with or without concurrent and/or adjuvant temozolomide in patients with non$1 p / 19 q$ deleted anaplastic gliomas (Figure 5). This type of dual study design allows for the patient populations to be enriched in a specific marker, yet it doesn't exclude either tumour types (codeleted and nondeleted $1 \mathrm{p} / 19 \mathrm{q}$ ). All specimens will also be tested for MGMT promoter methylation. 


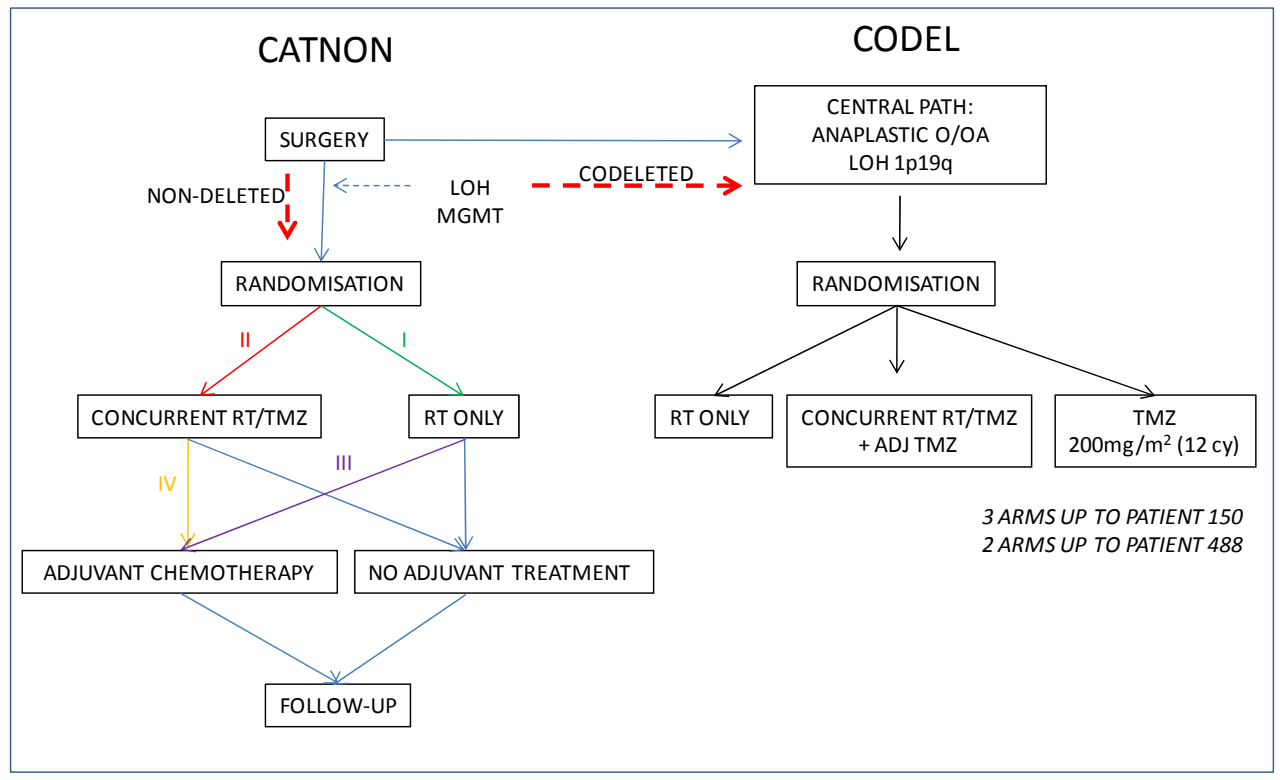

Fig. 5. Overview of the CATNON and CODEL trials

A phase I/IIa trial examined the effectiveness of adding cilengitide to concurrent chemoradiotherapy (Stupp et al. 2010). This study demonstrated the effectiveness of cilengitide but also showed that there was a clear survival benefit in the patients with MGMT promoter methylation (Stupp et al. 2010). The phase III CENTRIC trial (recruitment closed in Feb, 2011) restricted recruitment to newly diagnosed GBM patients with confirmed MGMT methylation. An additional two phase II trials sponsored by the pharmaceutical company, EMD Serono, are designed to treat patients with unmethylated MGMT: CORE (Cilengitide, Temozolomide, and Radiation Therapy in Treating Patients with Newly Diagnosed Glioblastoma and Unmethylated Gene Promoter Status) and ExCentric. CORE (trial still open; May 2011) examines the efficacy of increasing the dose schedule of cilengitide (2000mg twice weekly and $2000 \mathrm{mg}$ five times per week) versus standard concurrent chemoradiotherapy (without cilengitide). The ExCentric trial (recruitment open, May 2011) has taken a much different approach. In this trial, procarbazine is added to the concurrent schedule of radiotherapy, TMZ, cilengitide and patients will be treated adjuvantly for an additional 6 cycles with the triple cocktail of cilengitide, TMZ and procarbazine. The patients have so far shown excellent toleration of this combination.

The RTOG-0825 examines the effect of bevacizumab administered with radiotherapy compared to conventional concurrent chemoradiotherapy (TMZ) in primary GBM. All patients enrolled in this study will be tested for MGMT promoter methylation. Unique to this study, however, all samples will be prospectively tested with the nine-gene profile which separates the proneural GBM from the mesenchymal-angiogenic GBM (Colman et al. 2010). It is becoming mandatory for future trial design to incorporate molecular inclusion criteria to identify the poorly responding patients from the patients who benefit. 


\section{Targeted therapies for glioblastoma}

Molecular targeted therapies specifically inhibit amplified or aberrant proteins that drive tumour cell growth. The key to targeted therapy is identifying a target whose inhibition will stop the growth of the tumour cell. Whilst this field has rapidly developed, our understanding at the molecular level of the precise role that potential targets have in tumorigenesis and the survival dependence that tumours have on these components has not progressed at the same rate. Unlike melanoma, lung and breast cancer, glioblastoma lacks significant driver mutations which are present in ample abundance and in all tumours. The TCGA analysis revealed a wide spectrum of molecular variation in glioblastoma. TCGA used global gene expression analysis to show aberrations occurred more commonly in genes whose protein products regulated the core cell growth signalling pathways that were already known to be important such as EGFR, PTEN, p53 and CDKN2A. One pathway which is frequently dysregulated is the receptor tyrosine kinase (RTK)/phosphatidylinositol 3-kinase (PI3K)/Akt/mammalian target of rapamycin (mTOR) cascade. Approximately $86 \%$ of clinical samples analysed by the TCGA with both copy number and sequencing data had a genetic alteration in the RTK/PI3K pathway (Parsons et al. 2008). In addition, genetic alterations in two other core pathways; RB (87\%) and TP53 (78\%) were documented. At the time ( 3 years ago now) it was reasonable to suggest that all tumours be sequenced and the genetic aberrations be documented before selecting the targeted therapy. For example, for tumours with alterations in CDKN2A or CDKN2C or amplifications in CDK4 or CDK6, a CDK inhibitor should be recommended. Unfortunately, we underestimated the extent of genomic complexity and it is very doubtful that therapies targeted to a single genetic change will ever be effective. A range of molecular targeted drugs applied in combination or in addition to each other is needed (Jansen et al. 2010). In clinical practice, the multi-drug approach is currently limited by intellectual property. Most likely the efficacy of two drugs may require two competing pharmaceutical companies to work together.

To understand why our current single targeted therapies are ineffective, it is useful to examine the earlier clinical studies with EGFR- and VEGF-targeted drugs. We can also glean value from trials using targeted therapy in other cancers. Even when the target of interest is much more prominent such as KRAS or BRAF, valuable lessons can still be learnt.

\subsection{Targeting the RTK/PI3K pathway}

In a study of 49 patients with recurrent glioblastoma, tumour shrinkage was evident in 9 patients (25\%) (Mellinghoff et al. 2005). Logically, it was of great interest to better understand the underlying molecular biology of these 9 responders. Pre-treatment tissue was only available for 7 of the responding patients and 19 patients who did not respond. The authors found coexpression of EGFRvIII and PTEN sensitised glioblastoma to erlotinib and correctly validated this finding in tissue samples from different institutions undergoing similar treatment $(n=33)$ (Mellinghoff et al. 2005). Unfortunately, the relationship between EGFRvIII and intact PTEN co-expression did not translate to the subsequent prospective phase I/II trials (Brown et al. 2008; van den Bent et al. 2009). No relationship between aberrations in the RTK core and the EGFR inhibitor, lapatinib (Thiessen et al. 2010) or addition of erlotinib with the mTOR inhibitor, sirolimus (Reardon et al. 2010) were linked with response. 


\subsection{Targeting angiogenesis}

The development of anti-angiogenic agents for glioblastoma have been promising and include bevacizumab (Vascular endothelial growth factor [VEGF] antibody), cediranib (VEGF receptor antagonist), cilengitide (mentioned previously; integrin antagonist) and Enzastaurin (Protein Kinase-C- $\beta$-antagonist).

The preclinical and clinical data for cediranib treatment in glioblastoma looked very promising (Dietrich et al. 2009; Gerstner et al. 2011). Unfortunately, the International Multicentre Phase III trial, REGAL was negative. The REGAL study compared the use of cediranib alone, cediranib in combination with lomustine and lomustine plus placebo. In the 325 patients with recurrent GBM studied, only $16 \%$ treated with cediranib monotherapy were alive and progression free at 6 months (APF6) compared to $34.5 \%$ in the combination group and $24.5 \%$ in the lomustine plus placebo group (results reported by T. Batchelor at the Society of Neuro-oncology Annual Meeting, 2010; (Ahluwalia 2011)). Akin to cediranib, preclinical and studies of enzastaurin showed good anti-glioma activity but failed to show any significant benefits when trialled in a phase III study comparing enzastaurin to lomustine. Although less toxicity was observed with enzastaurin, no significant differences in median progression free survival and overall survival were observed (Wick et al. 2011). The humanized antibody, Bevacizumab (Avastin), has received the most attention, with Food and Drug Administration (FDA) approval for use in recurrent GBM in the USA. No such approvals have been obtained in Europe and Australia. This is predominantly because there is only a modest overall survival benefit of 7.8-9.2 months suggesting a further improvement of efficacy is needed. Numerous phase II studies have shown modest survival benefits with bevacizumab either as a monotherapy or in combination with irinotecan (Chinot et al. 2011; Jakobsen et al. 2011; Lai et al. 2011; Prados et al. 2011; Reardon et al. 2011). Consistent to all trials examining bevacizumab efficacy is the reduction of steroids for patients and valuable palliation with preservation of key performance status (KPS), supporting a role for bevacizumab as a therapy in late stage disease (Hofer et al. 2011). Whether bevacizumab results in true glioma cell destruction or is it merely its ability to control the perivascular leak, resulting in better symptom control (associated with improvement of gadolinium MRI) needs to be elucidated.

An issue consistent with all trials of cediranib, enzastaurin and bevacizumab is their testing on recurrent glioblastoma as opposed to primary glioblastoma. Recurrent glioblastoma are already highly refractory to treatment and the potential benefits of these drugs may be missed. New studies are investigating bevacizumab up front with standard radiation therapy and temozolomide. This has shown to be well tolerated (Vredenburgh et al. 2011) and it is a strategy that the RTOG-0825 trial has incorporated (discussed previously).

With all of these targeted therapies, it would seem obvious that the more target present, the more efficacious the drug. Unfortunately, this has not been the case. For example, why patients with high expression of VEGF have not shown strong response to bevacizumab? These issues pertaining to biomarkers in targeted therapy trials will be discussed in turn below:

\subsection{Many retrospective analyses of single arm investigations are performed in small and often heterogeneous cohorts of patients}

The co-expression of EGFRvIII and PTEN was first discovered in an initial test set consisting of 49 recurrent GBM treated with either gefitinib $(n=37)$ or erlotinib $(n=12) .26$ patients with 
clear-cut evidence of a response or tumour progression had sufficient tissue for molecular analysis. Hence, just over half of the originally small cohort was analysed for molecular biomarkers. The validation study used a different tissue type entirely as only paraffinembedded slides were available. Again, this material was untreated tumour tissue, not the recurrent lesion. The validation set was extremely underpowered $(n=33)$ with only 8 responders identified in this dataset. It is imperative that collaborations between different institutes and countries work together to increase the power of these biomarker studies.

\subsubsection{A lack of standardisation in the methods used for marker measurement}

Assays for biomarkers need to be reliable. The assay needs to give identical results if repeated in the same or in another laboratory. The result needs to be the same, even when different methodologies are used. And finally, we need to ask whether the test provides added value to clinical practice. This has often been a strong criticism of studies incorporating MGMT promoter methylation (as discussed previously) and unfortunately the same issues surround biomarkers for targeted therapies. The original study by Mellinghoff and colleagues used immunohistochemistry (IHC) to assess PTEN expression (Mellinghoff et al. 2005). The problem with this approach is the antibody used does not detect the full length PTEN protein. Should mutations arise in the C-terminal end of the protein, these would go undiscovered using IHC assay. IHC for EGFR is also contentious. EGFR overexpression in GBM is generally driven by EGFR amplification. The scoring of EGFR IHC can be variable and different antibodies have different specificities to the EGFR protein. Amplification of EGFR or more specifically gain of copy number is most commonly detected by fluorescence in situ hybridization (FISH) and can be routinely performed in most histopathological laboratories. What is puzzling is the lack of sequencing of both EGFR and PTEN genes in the subsequent phase I/II clinical trials assessing TKIs. The most frequent mutant form of EGFR is EGFR Variant III (EGFRvIII or EGFR delta) which is missing the ligand -binding domain resulting in the constitutive activation of the EGFRphosphoinositide 3-kinase pathway. IHC specific to the EGFRvIII mutant form is highly specific as too is the commonly used RT-PCR method. However, there are additional missense mutations encoding extracellular EGFR that have been shown to drive oncogenesis in vitro and can be inhibited by small-molecular tyrosine kinase inhibitors.

The original pre-clinical/clinical study sequenced all exons and flanking intronic sequences for EGFR (kinase domain), the HER2/neu (kinase domain) and all exons of PTEN. FISH was also performed to detect EGFR amplification and RT-PCR was used to amplify EGFR (1044bp product) and EGFRvIII (243-bp product). In addition, EGFR and PTEN were examined with IHC (Mellinghoff et al. 2005). 26 of the 49 patients underwent sequencing, which included 6 patients who showed a response to erlotinib. No mutations were found. Van den Bent and colleagues assessed the benefits of erlotinib compared to temozolomide or cumustine in recurrent GBM in a randomized phase II study (van den Bent et al. 2009). Obtaining full data for all patients in this study was problematic. From 100 patients, PTEN expression could be determined in 82 patient cases and pAKT in 64 patients. Like the Mellinghoff study, no mutations in EGFR were detected, however only exons 19 to 21 were assessed. Although an association between EGFRvIII and EGFR amplification with poor overall survival was shown, no correlation between response and the co-expression of PTEN and EGFR was measured (van den Bent et al. 2009). In fact, no significant activity of erlotinib was observed. In another study of 65 patients, erlotinib efficacy was assessed in 
combination with temozolomide (Prados et al. 2009). Again no association with EGFRvIII and PTEN and response was measured, however in this study, MGMT promoter methylation was associated with better response. EGFR was measured with FISH and IHC, PTEN and EGFRvIII were analysed by IHC. No mutational analysis of EGFR was undertaken. Reardon and colleagues assessed the combination of erlotinib with a mTOR inhibitor, sirolimus in recurrent GBM (Reardon et al. 2010). Again, EGFR, EGFRvIII, PTEN, PI3K and pS6 were assessed by IHC and no association for these markers with clinical response was found. Mutational analysis was not conducted. Moreover, the general methodologies did not differ in the studies addressing erlotinib and response and the Phase II studies could not validate the findings of Mellinghoff et al.

Elegant biomarker studies have been associated with the anti-angiogenic drugs. Attention has focused predominantly on secreted factors and imaging modalities. Interleukin 6 (IL-6) is over-expressed in the majority of gliomas and functions as an immune regulator and an autocrine growth factor (Saidi et al. 2009). High starting levels of IL-6 may influence the efficacy of bevacizumab as it provides redundancy for the VEGF/VEGFR pathway and promotes an immune response that stimulates angiogenesis by non-VEGF mechanisms. Sorenson et al. reported the combination of MRI imaging (measured changes in vascular permeability/flow [Ktrans] and changes in microvessel volume) and circulating collagen IV levels in plasma to be predictive of outcome in glioblastoma patients treated with cediranib (Sorensen et al. 2009). The level of circulating endothelial progenitor cells (cEPCs) and viable circulating endothelial cells (cECs) has also been shown to correlate with response (Sorensen et al. 2009). The ability to identify changes in a tumour's perfusion offers the potential to predict growth or regression. Dynamic susceptibility-weighted contrast-enhanced (DSC) MR imaging can be used to measure relative cerebral blood volume (rCBV) as a surrogate marker of perfusion. A pilot study of 16 patients with recurrent glioblastoma and treatment with bevacizumab found that MR perfusion imaging showed a significantly improved correlation with time to progression (Sawlani et al. 2011). Studies from Tsien (Tsien et al. 2011) and Server (Server et al. 2011)- both show positive results for this scan in patients with PsPD. Only changes in the hypoxia inducing factor (HIF) 2 alpha [HIF2 $\alpha$ ] have been shown to be promising surrogates of response to anti-angiogenic therapies (Mao et al. 2011).

\subsubsection{Methodologies chosen in the study may not represent a comprehensive analysis of multiple components of a specific pathway}

None of the studies examining erlotinib have comprehensively analysed the downstream components involved in EGFR signalling. Additional testing of PI3K and PS6 were added in some studies. It is very common for glioblastomas to have dysregulated signalling cascades downstream of EGFR, particular the negative feedback loops. Several growth factor pathways are also triggered. It's not economically feasible in most instances to assess all aspects of the RTK/PI3K/AKT/mTOR signalling cascade. However, a new system of testing drugs and identifying which subtypes of glioblastoma are susceptible to the drug could be to use human glioblastoma xenograft panels serially passaged in nude mice. This model allows tumour burden to be monitored non-invasively and rapid assessment of biological pathways (Prasad et al. 2011).

Feedback mechanisms also pose an issue with targeted therapies blocking angiogenesis. Tumours frequently recur after treatment with cediranib and bevacizumab and are refractory to further treatments. There have been different theories postulated as to why this "rebound" effect occurs. Tumours may switch to VEGF-independent angiogenic pathways 
or vessel co-option. A commonly held theory is that recurrent glioblastomas switch their growth pattern after anti-VEGF treatment (di Tomaso et al. 2011). The tumour cells are exposed to an increased hypoxic environment leading to increased migration, invasion, heightened glycolysis and increased PI3K pathway activation. Combining bevacizumab with anti-glycolytic agents or PI3K inhibitors might be more effective. Tumour-initiating CD133+ve cells are radio-resistant and can self renew to reform tumours, suggesting that these cells are responsible for tumour relapse (Liu et al. 2009). More significantly, exposure to bevacizumab inhibited the maturation of tumour endothelial progenitors into the endothelium but not the differentiation of CD133+ cells into progenitor cells (Wang et al. 2011). This fundamental study showed that there is a dynamic balance between the CD133+ cell population and tumour cells and we need to target the endothelial transition as well as VEGF.

\subsubsection{Not all mutations within a given gene are screened}

In simplistic terms, the plethora of TKIs are designed to be effective on patients harbouring EGFR mutations. However, in the majority of studies exploring gefitinib and erlotinib, the EGFR gene is not fully sequenced to identify variants and mutations. TCGA analyses have identified a high diversity of genes mutated within glioblastoma. As prices drop with Next Generation sequencing, capabilities to better define precise genetic aberrations associated with response to a specific treatment will improve. Copy number aberrations (amplifications and deletions) and structural aberrations (intra-chromosomal rearrangements- inversions, inverted/tandem duplications) are not detected using traditional Sanger sequencing in the lab. Our ability to assess these aberrations must improve at the rate that new targeted therapies are flooding the market. BRAF is a commonly deleted gene in approximately $8 \%$ of solid tumours, however over 30 different mutations in the BRAF gene have been implicated in cancer (Dienstmann\&Tabernero 2011; Puzanov et al. 2011).

\subsubsection{A pathway-centric approach is needed}

As eluded to in our discussion of multiple pathways and feedback loops in any given target, we need to develop ways to target multiple points of a pathway akin to attacking the Achilles heel of the tumour. Recent data suggest that miRNA expression is tightly coordinated, and that each miRNA may target numerous messages. Thus, a specific miRNA has the potential to regulate several members of an entire signalling pathway. miRNAs negatively regulate their targets by one of two mechanisms: either by near perfect binding to the mRNA target and induction of miRNA-associated, multiprotein RNA-induced-silencing complex (miRISC), which results in accelerated mRNA decay (Yekta 2004; Wu 2006) or by less perfect binding to the target mRNA 3'-UTR and inhibition of translation through a RISC complex similar to, or identical with, the complex recruited in RNA interference (RNAi) (Humphreys et al. 2005; Pillai et al. 2005; Esquela-Kerscher\&Slack 2006).

miR-7 directly regulates the expression of EGFR in glioblastoma and has also been shown to directly attenuate the activation of AKT and ERK1/2 (extracellular signal-regulated kinase) indicating its ability to co-ordinately regulate EGFR signalling (Webster et al. 2009). We also showed that miR-124a attenuated glioblastoma migration and invasion at multiple points of the pathway (Fowler et al. 2011). New technologies are currently being developed to facilitate the use of miRNAs as a realistic therapeutic option. Until then, combination treatments and developing inhibitors which can affect a multiplicity of targets are critical. 


\subsubsection{Differing response criterion}

The inability to accurately define endpoints from clinical trials makes the evaluation of new therapies subjective and significantly delays treatment development. At present overall survival (OS) and 6 month progression free survival (PFS6) are two defined end points accepted in most clinical trials testing for new GBM therapies. PFS6 relies on a combination of gadolinium enhanced MRI imaging and potentially subjective clinical evaluation. Seizures, depression and steroid induced myopathy can all influence clinical signs and symptoms. Since 1990, the MacDonald criterion has been used as an objective radiologic assessment of response in GBM. This two dimensional measurement has been mainstay for evaluating tumour response and is based upon measurements of the enhancing tumour area (the product of the maximal cross-sectional enhancing diameters) (Macdonald et al. 1990). With the advance of treatments administered to patients with GBM, the MacDonald Criteria has a number of important limitations. The MacDonald criteria does not discriminate measurable disease from non-measureable disease, cannot identify non-tumour related increases in enhancement and provides no use for the evaluation of anti-angiogenic drugs. Bevacizumab can cause accelerated regression of VEGF driven angiogenesis and rapid resolution of gadolinium MRI changes in responding patients. There is concern however as to whether anti-VEGF therapy results in true glioma cell destruction or their ability to control the perivascular leak, resulting in better symptom control (associated with improvement of gadolinium MRI).

An international working group was formed to review and improve the response assessment criteria for high grade gliomas, coined Response Assessment in Neuro-Oncology (RANO) (Wen et al. 2010). The guidelines have devised a better standardisation of how clinicians measure response, which will ultimately result in a more uniform assessment of disease status across different centres. Unfortunately, the new RANO guidelines do not address the persistent problem of the irregularity of gliomas and the difficulty of measuring tumours treated with anti-angiogenic drugs, suggesting that volumetric measurements that count all enhancing and non-enhancing voxels may prove more accurate in the future.The RANO working party acknowledges that an important area of future research is the need to develop advanced novel MRI techniques.

\subsubsection{Inadequate tissue}

Biobanks or biorepositories play a critical role in the evolution of biomarkers, targets and targeted therapies. Five years ago, the NCI announced their plans to enlist dozens of biorepositories in the USA to provide large tumour numbers and use high-throughput DNA sequencing and computational biology to produce with new methods of detecting and treating cancers. Unfortunately sub-standard tissue and data collection provided a significant road block to the Cancer Genome Atlas effort. Biorepositories remain underfunded and unappreciated. Despite billions of dollars poured into cancer research, innovation in the field of biobanking is sadly lacking. Standard operating procedures (SOPs) are not consistent between sites, and sometimes differ within single institutes. Methodologies for preserving tissue vary and times between tumour removal and time of processing fluctuate. Significant genetic changes can occur between the time of tissue removal from the body and time of processing. The collection of tissue has to be taken seriously and investments need to be urgently made to promote basic, translational and clinical research as well as social gain in terms of improved cancer care and economic development. 
Collection and storage of frozen tissue is critical for biomarker development. Many of our current biomarker assays are performed on Formalin Fixed Paraffin Embedded (FFPE) tissue. This type of tissue, whilst preserving morphology for diagnosis, induces problems for downstream molecular applications. High quality RNA is difficult to obtain from FFPE tissue and PCR amplification from FFPE DNA is limited to products of less than 200 base pairs. It is also difficult to control the processes leading up to tissue fixation. In a first class Neuro-oncology centre in Australia, FFPE blocks were being sent to Central Headquarters for MGMT methylation detection. Unfortunately, a sizeable batch of tissues were nondeterminative (could not be amplified). Tissue from surgeries performed on a Friday were fixed in formalin, however the laboratory was unattended over the weekend, resulting in the tissue submerged in formalin for up to 72 hours (routinely, formalin should be removed after 24 hours).

Another issue that we are not taking into careful consideration are the molecular changes acquired in the tumour after treatment. Many biomarker studies are performed on tumour obtained at initial surgery event. This tissue has not been exposed to treatment. However, the majority of novel treatments are tested at the time of recurrence. Changes in chromosome aberrations and mismatch repair proteins have been detected in paired tumour specimens (primary and relapsed). Careful consideration of the tissue and its relevance to the clinical circumstance of the patient is required.

\section{Future directions for biomarker development}

To advance personalised medicine, a co-operative effort between cancer researchers and clinicians is urgently needed. There is very little collaboration between scientists working on targeted therapies such as the TKIs and anti-angiogenics....what worked, what didn't? Specific consideration needs to be paid to increasing sample sizes, sequencing entire genes, implementing robust methodologies and taking a holistic approach to understanding pathways. Cancer is multifaceted and we urgently need to unravel these complexities. Two prospective biomarker trials have been encouraging: the I-SPY 2 (investigation of serial studies to predict your therapeutic response with imaging and molecular analysis 2) for women with locally advanced breast cancer (Barker et al. 2009) and BATTLE (Biomarker Integrated Approaches of Targeted Therapy for Lung Cancer Elimination) for pre-treated patients with non-small cell lung cancer (NSLC) (Kim 2011). Both trials employ an adaptive phase II/III clinical trial design. The I-SPY 2 is performed as a neo-adjuvant trial. A core biopsy is provided and tested for Estrogen Receptor (ER), Progesterone Receptor (PR), Human Epidermal Growth Factor Receptor 2 (HER2) and MammaPrint status (a gene signature known to be predictive of outcome). Based upon the marker outcomes, the patients will be stratified into two arms of a standard neoadjuvant regime: paclitaxel (plus trastuzumab [Herceptin] for HER2+ patients followed by doxorubicin (Adriamycin) and cyclophosphamide (Cytoxan). Five new drugs will be trialled in the other arms (each being added to the standard therapy). Patients are currently being recruited. The BATTLE trial takes on a very similar adaptive design but differs in its examination of samples from posttreated NSLC. Key drugs and associated biomarkers (Erlotinib/EGFR; Vandetanib/VEGFR; Erlotinib + bexarotene/ Retinoid + EGFR and Sorafenib/ KRAS/BRAF) were tested both as an equal randomisation design and an adaptive randomisation design. This trial confirmed that tumours harbouring mutations in KRAS/BRAF showed a disease control of $79 \%$ when treated with sorafenib but only $14 \%$ of the patients responded to erlotinib. Conversely, 
sorafenib, although active against wild type or mutated KRAS, had worse disease control in patients with EGFR mutations. A limitation in applying these adaptive trial designs to glioblastoma will be adequate numbers. Additionally, the BATTLE trial identified that grouping mutations together were less predictive than individual markers. This may also impact on a highly heterogenous cancer such as glioblastoma. Nevertheless, these trials show that, with a highly integrated team of multidisciplinary investigators, better overall survival in glioblastoma is achievable.

\section{Acknowledgements}

I would like to acknowledge the support and financial assistance from the Cure For Life Foundation and the Cancer Institute NSW.

\section{References}

(2008). Comprehensive genomic characterization defines human glioblastoma genes and core pathways. Nature, Vol. 455, No. 7216, (Oct 23) pp. 1061-1068.

Ahluwalia, M. S. (2011). 2010 Society for Neuro-Oncology Annual Meeting: a report of selected studies. Expert Rev Anticancer Ther, Vol. 11, No. 2, (Feb) pp. 161-163.

Balss, J., et al. (2008). Analysis of the IDH1 codon 132 mutation in brain tumors. Acta Neuropathol, Vol. 116, No. 6, (Dec) pp. 597-602.

Barbashina, V., et al. (2005). Allelic losses at 1p36 and 19q13 in gliomas: correlation with histologic classification, definition of a $150-\mathrm{kb}$ minimal deleted region on 1p36, and evaluation of CAMTA1 as a candidate tumor suppressor gene. Clin Cancer Res, Vol. 11, No. 3, (Feb 1) pp. 1119-1128.

Barker, A. D., et al. (2009). I-SPY 2: an adaptive breast cancer trial design in the setting of neoadjuvant chemotherapy. Clin Pharmacol Ther, Vol. 86, No. 1, (Jul) pp. 97-100.

Brandes, A. A., et al. (2001). Changing boundaries in the treatment of malignant gliomas. Expert Rev Anticancer Ther, Vol. 1, No. 3, (Oct) pp. 357-370.

Brandes, A. A., et al. (2006). Temozolomide 3 weeks on and 1 week off as first-line therapy for recurrent glioblastoma: phase II study from gruppo italiano cooperativo di neuro-oncologia (GICNO). Br J Cancer, Vol. 95, No. 9, (Nov 6) pp. 1155-1160.

Brell, M., et al. (2005). Prognostic significance of O6-methylguanine-DNA methyltransferase determined by promoter hypermethylation and immunohistochemical expression in anaplastic gliomas. Clin Cancer Res, Vol. 11, No. 14, (Jul 15) pp. 5167-5174.

Brock, C. S., et al. (1998). Phase I trial of temozolomide using an extended continuous oral schedule. Cancer Research, Vol. 58, No. 19, (Oct 1) pp. 4363-4367.

Brown, P. D., et al. (2008). Phase I/II trial of erlotinib and temozolomide with radiation therapy in the treatment of newly diagnosed glioblastoma multiforme: North Central Cancer Treatment Group Study N0177. J Clin Oncol, Vol. 26, No. 34, (Dec 1) pp. 5603-5609.

Buttolo, L., et al. (2006). Alternative schedules of adjuvant temozolomide in glioblastoma multiforme: A 6-year experience. Journal Of Clinical Oncology, Vol. 24, No. 18, (Jun 20) pp. 60S-60S.

Cairncross, G., et al. (2006). Phase III trial of chemotherapy plus radiotherapy compared with radiotherapy alone for pure and mixed anaplastic oligodendroglioma: 
Intergroup Radiation Therapy Oncology Group Trial 9402. J Clin Oncol, Vol. 24, No. 18, (Jun 20) pp. 2707-2714.

Calin, G. A., et al. (2002). Frequent deletions and down-regulation of micro- RNA genes miR15 and miR16 at 13q14 in chronic lymphocytic leukemia. Proc Natl Acad Sci U S A, Vol. 99, No. 24, (Nov 26) pp. 15524-15529.

Cao, V. T., et al. (2009). The correlation and prognostic significance of MGMT promoter methylation and MGMT protein in glioblastomas. Neurosurgery, Vol. 65, No. 5, (Nov) pp. 866-875; discussion 875.

Chinot, O. L., et al. (2011). AVAglio: Phase 3 trial of bevacizumab plus temozolomide and radiotherapy in newly diagnosed glioblastoma multiforme. Adv Ther, Vol. 28, No. 4, (Apr) pp. 334-340.

Christensen, B. C., et al. (2011). DNA methylation, isocitrate dehydrogenase mutation, and survival in glioma. J Natl Cancer Inst, Vol. 103, No. 2, (Jan 19) pp. 143-153.

Colman, H., et al. (2010). A multigene predictor of outcome in glioblastoma. Neuro Oncol, Vol. 12, No. 1, (Jan) pp. 49-57.

Conti, A., et al. (2009). miR-21 and 221 upregulation and miR-181b downregulation in human grade II-IV astrocytic tumors. J Neurooncol, Vol. 93, No. 3, (Jul) pp. 325-332.

Dang, L., et al. (2009). Cancer-associated IDH1 mutations produce 2-hydroxyglutarate. Nature, Vol. 462, No. 7274, (Dec 10) pp. 739-744.

de Souza, J. A. and O. I. Olopade (2011). CYP2D6 genotyping and tamoxifen: an unfinished story in the quest for personalized medicine. Semin Oncol, Vol. 38, No. 2, (Apr) pp. 263-273.

di Tomaso, E., et al. (2011). Glioblastoma recurrence after cediranib therapy in patients: lack of "rebound" revascularization as mode of escape. Cancer Res, Vol. 71, No. 1, (Jan 1) pp. 19-28.

Dienstmann, R. and J. Tabernero (2011). BRAF as a Target for Cancer Therapy. Anticancer Agents Med Chem, Vol. 11, No. 3, (Mar 1) pp. 285-295.

Dietrich, J., et al. (2009). Cediranib: profile of a novel anti-angiogenic agent in patients with glioblastoma. Expert Opin Investig Drugs, Vol. 18, No. 10, (Oct) pp. 1549-1557.

Dresemann, G., et al. (2010). Imatinib in combination with hydroxyurea versus hydroxyurea alone as oral therapy in patients with progressive pretreated glioblastoma resistant to standard dose temozolomide. J Neurooncol, Vol. 96, No. 3, (Feb) pp. 393-402.

Ducray, F., et al. (2009). alpha-Internexin expression identifies $1 \mathrm{p} 19 \mathrm{q}$ codeleted gliomas. Neurology, Vol. 72, No. 2, (Jan 13) pp. 156-161.

Ducray, F., et al. (2008). Anaplastic oligodendrogliomas with $1 \mathrm{p} 19 \mathrm{q}$ codeletion have a proneural gene expression profile. Mol Cancer, Vol. 7, No., pp. 41.

Esquela-Kerscher, A. and F. J. Slack (2006). Oncomirs - microRNAs with a role in cancer. Nat Rev Cancer, Vol. 6, No. 4, (Apr) pp. 259-269.

Esteller, M., et al. (2000). Inactivation of the DNA-repair gene MGMT and the clinical response of gliomas to alkylating agents. N Engl J Med, Vol. 343, No. 19, (Nov 9) pp. 1350-1354.

Esteller, M., et al. (1999). Inactivation of the DNA repair gene O6-methylguanine-DNA methyltransferase by promoter hypermethylation is a common event in primary human neoplasia. Cancer Res, Vol. 59, No. 4, (Feb 15) pp. 793-797.

Fowler, A., et al. (2011). miR-124a is frequently down-regulated in glioblastoma and is involved in migration and invasion. Eur J Cancer, Vol. 47, No. 6, (Apr) pp. 953-963. 
Fuller, G. N. and B. W. Scheithauer (2007). The 2007 Revised World Health Organization (WHO) Classification of Tumours of the Central Nervous System: newly codified entities. Brain Pathol, Vol. 17, No. 3, (Jul) pp. 304-307.

Gan, H. K., et al. (2009). The EGFRvIII variant in glioblastoma multiforme. J Clin Neurosci, Vol. 16, No. 6, (Jun) pp. 748-754.

Gerson, S. L. (2004). MGMT: its role in cancer aetiology and cancer therapeutics. Nat Rev Cancer, Vol. 4, No. 4, (Apr) pp. 296-307.

Gerstner, E. R., et al. (2011). Infiltrative patterns of glioblastoma spread detected via diffusion MRI after treatment with cediranib. Neuro Oncol, Vol. 12, No. 5, (May) pp. 466-472.

Goetz, M. P. (2010). Update on CYP2D6 and its impact on tamoxifen therapy. Clin Adv Hematol Oncol, Vol. 8, No. 8, (Aug) pp. 536-538.

Hartmann, C., et al. (2010). Patients with IDH1 wild type anaplastic astrocytomas exhibit worse prognosis than IDH1-mutated glioblastomas, and IDH1 mutation status accounts for the unfavorable prognostic effect of higher age: implications for classification of gliomas. Acta Neuropathol, Vol. 120, No. 6, (Dec) pp. 707-718.

Hawkins, N. J., et al. (2009). MGMT methylation is associated primarily with the germline C>T SNP (rs16906252) in colorectal cancer and normal colonic mucosa. Mod Pathol, Vol. 22, No. 12, (Dec) pp. 1588-1599.

Hegi, M. E., et al. (2005). MGMT gene silencing and benefit from temozolomide in glioblastoma. N Engl J Med, Vol. 352, No. 10, (Mar 10) pp. 997-1003.

Hill, C., et al. (2003). Genetic markers in glioblastoma: prognostic significance and future therapeutic implications. Adv Anat Pathol, Vol. 10, No. 4, (Jul) pp. 212-217.

Hofer, S., et al. (2011). Clinical outcome with bevacizumab in patients with recurrent highgrade glioma treated outside clinical trials. Acta Oncol, Vol. 50, No. 5, (Jun) pp. 630635.

Hormigo, A., et al. (2006). YKL-40 and matrix metalloproteinase-9 as potential serum biomarkers for patients with high-grade gliomas. Clin Cancer Res, Vol. 12, No. 19, (Oct 1) pp. 5698-5704.

$\mathrm{Hu}, \mathrm{J}$. C. and K. Mokbel (2001). Does c-erbB2/HER2 overexpression predict adjuvant tamoxifen failure in patients with early breast cancer? Eur J Surg Oncol, Vol. 27, No. 4, (Jun) pp. 335-337.

Humphreys, D. T., et al. (2005). MicroRNAs control translation initiation by inhibiting eukaryotic initiation factor 4E/cap and poly(A) tail function. Proc Natl Acad Sci U S A, Vol. 102, No. 47, (Nov 22) pp. 16961-16966.

Inda, M. M., et al. (2010). Tumor heterogeneity is an active process maintained by a mutant EGFR-induced cytokine circuit in glioblastoma. Genes Dev, Vol. 24, No. 16, (Aug 15) pp. 1731-1745.

Jakobsen, J. N., et al. (2011). Irinotecan and bevacizumab in recurrent glioblastoma multiforme. Expert Opin Pharmacother, Vol. 12, No. 5, (Apr) pp. 825-833.

Jansen, M., et al. (2010). Molecular pathology in adult gliomas: diagnostic, prognostic, and predictive markers. Lancet Neurol, Vol. 9, No. 7, (Jul) pp. 717-726.

Jenkins, R. B., et al. (2006). A t $(1 ; 19)(q 10 ; p 10)$ mediates the combined deletions of $1 p$ and 19q and predicts a better prognosis of patients with oligodendroglioma. Cancer Res, Vol. 66, No. 20, (Oct 15) pp. 9852-9861.

Ji, J., et al. (2009). MicroRNA expression, survival, and response to interferon in liver cancer. N Engl J Med, Vol. 361, No. 15, (Oct 8) pp. 1437-1447. 
Kaloshi, G., et al. (2007). FABP7 expression in glioblastomas: relation to prognosis, invasion and EGFR status. J Neurooncol, Vol. 84, No. 3, (Sep) pp. 245-248.

Kamiryo, T., et al. (2004). Correlation between promoter hypermethylation of the O6methylguanine-deoxyribonucleic acid methyltransferase gene and prognosis in patients with high-grade astrocytic tumors treated with surgery, radiotherapy, and 1-(4-amino-2-methyl-5-pyrimidinyl)methyl-3-(2-chloroethyl)-3-nitrosourea-b ased chemotherapy. Neurosurgery, Vol. 54, No. 2, (Feb) pp. 349-357; discussion 357.

Karayan-Tapon, L., et al. (2010). Prognostic value of O6-methylguanine-DNA methyltransferase status in glioblastoma patients, assessed by five different methods. Journal of neuro-oncology, Vol. 97, No. 3, pp. 311-322.

Kefas, B., et al. (2009). The neuronal microRNA miR-326 acts in a feedback loop with notch and has therapeutic potential against brain tumors. J Neurosci, Vol. 29, No. 48, (Dec 2) pp. 15161-15168.

Kim, E., Herbst RS, Wistuba II, Lee IJ, Blumenschein GR, Tsao A, Stewart DJ, Hicks ME, Erasmus J, Gupta S, Alden CM, Liu S, Tang X, Khuri FR, Tran HT, Johnson BE, Heymach JV, Mao L, Fossella F, Kies MS, Papadimitrakopoulou, David SE, Lippman SM, Hong WK (2011). The BATTLE Trial: Personalizing Therapy for Lung Cancer. Cancer Discovery, Vol. 1, No. 1, pp. 43.

Kim, H., et al. Integrative genome analysis reveals an oncomir/oncogene cluster regulating glioblastoma survivorship. Proc Natl Acad Sci U S A, Vol. 107, No. 5, (Feb 2) pp. 2183-2188.

Kim, H. P., et al. (2008). Combined lapatinib and cetuximab enhance cytotoxicity against gefitinib-resistant lung cancer cells. Mol Cancer Ther, Vol. 7, No. 3, (Mar) pp. 607615.

Koch, D., et al. (2007). Local intracerebral administration of $\mathrm{O}(6)$-benzylguanine combined with systemic chemotherapy with temozolomide of a patient suffering from a recurrent glioblastoma. J Neurooncol, Vol. 82, No. 1, (Mar) pp. 85-89.

Kurokawa, H., et al. (2000). Inhibition of HER2/neu (erbB-2) and mitogen-activated protein kinases enhances tamoxifen action against HER2-overexpressing, tamoxifenresistant breast cancer cells. Cancer Res, Vol. 60, No. 20, (Oct 15) pp. 5887-5894.

Lai, A., et al. (2011). Phase II study of bevacizumab plus temozolomide during and after radiation therapy for patients with newly diagnosed glioblastoma multiforme. $J$ Clin Oncol, Vol. 29, No. 2, (Jan 10) pp. 142-148.

Laigle-Donadey, F., et al. (2005). [Molecular biology of oligodendroglial tumors]. Neurochirurgie, Vol. 51, No. 3-4 Pt 2, (Sep) pp. 260-268.

Layfield, L. J., et al. (2006). Epidermal growth factor receptor gene amplification and protein expression in glioblastoma multiforme: prognostic significance and relationship to other prognostic factors. Appl Immunohistochem Mol Morphol, Vol. 14, No. 1, (Mar) pp. 91-96.

Li, Y., et al. (2009). MicroRNA-21 targets LRRFIP1 and contributes to VM-26 resistance in glioblastoma multiforme. Brain Res, Vol. 1286, No., (Aug 25) pp. 13-18.

Liu, Q., et al. (2009). Molecular properties of CD133+ glioblastoma stem cells derived from treatment-refractory recurrent brain tumors. J Neurooncol, Vol. 94, No. 1, (Aug) pp. $1-19$.

Macdonald, D. R., et al. (1990). Response criteria for phase II studies of supratentorial malignant glioma. J Clin Oncol, Vol. 8, No. 7, (Jul) pp. 1277-1280. 
Mao, X. G., et al. (2011). Overexpression of ZNF217 in glioblastoma contributes to the maintenance of glioma stem cells regulated by hypoxia-inducible factors. Lab Invest, Vol., No., (Apr 11).

Mellai, M., et al. (2009). MGMT promoter hypermethylation in a series of 104 glioblastomas. Cancer Genomics Proteomics, Vol. 6, No. 4, (Jul-Aug) pp. 219-227.

Mellinghoff, I. K., et al. (2005). Molecular determinants of the response of glioblastomas to EGFR kinase inhibitors. N Engl J Med, Vol. 353, No. 19, (Nov 10) pp. 2012-2024.

Mikkelsen, T., et al. (2009). Temozolomide single-agent chemotherapy for newly diagnosed anaplastic oligodendroglioma. Journal of neuro-oncology, Vol. 92, No. 1, pp. 57-63.

Mokhtari, K., et al. (2011). Alpha-internexin expression predicts outcome in anaplastic oligodendroglial tumors and may positively impact the efficacy of chemotherapy: European organization for research and treatment of cancer trial 26951. Cancer, Vol., No., (Jan 18).

Mukasa, A., et al. (2004). Selective expression of a subset of neuronal genes in oligodendroglioma with chromosome 1p loss. Brain Pathol, Vol. 14, No. 1, (Jan) pp. 34-42.

Mukasa, A., et al. (2002). Distinction in gene expression profiles of oligodendrogliomas with and without allelic loss of 1p. Oncogene, Vol. 21, No. 25, (Jun 6) pp. 3961-3968.

Nakamura, M., et al. (2001). Promoter methylation of the DNA repair gene MGMT in astrocytomas is frequently associated with G:C --> A:T mutations of the TP53 tumor suppressor gene. Carcinogenesis, Vol. 22, No. 10, (Oct) pp. 1715-1719.

Newlands, E. S., et al. (2003). Phase I study of temozolamide (TMZ) combined with procarbazine (PCB) in patients with gliomas. Br J Cancer, Vol. 89, No. 2, (Jul 21) pp. 248-251.

Noushmehr, H., et al. (2010). Identification of a CpG island methylator phenotype that defines a distinct subgroup of glioma. Cancer Cell, Vol. 17, No. 5, (May 18) pp. 510522.

Nutt, C. L., et al. (2003). Gene expression-based classification of malignant gliomas correlates better with survival than histological classification. Cancer Res, Vol. 63, No. 7, (Apr 1) pp. 1602-1607.

Parsa, A. T., et al. (2007). Loss of tumor suppressor PTEN function increases B7-H1 expression and immunoresistance in glioma. Nat Med, Vol. 13, No. 1, (Jan) pp. 8488.

Parsons, D. W., et al. (2008). An integrated genomic analysis of human glioblastoma multiforme. Science, Vol. 321, No. 5897, (Sep 26) pp. 1807-1812.

Paz-Ares, L., et al. (2010). Clinical outcomes in non-small-cell lung cancer patients with EGFR mutations: pooled analysis. J Cell Mol Med, Vol. 14, No. 1-2, (Jan) pp. 51-69.

Paz, M. F., et al. (2004). CpG island hypermethylation of the DNA repair enzyme methyltransferase predicts response to temozolomide in primary gliomas. Clin Cancer Res, Vol. 10, No. 15, (Aug 1) pp. 4933-4938.

Pegg, A. E. (1990). Mammalian O6-alkylguanine-DNA alkyltransferase: regulation and importance in response to alkylating carcinogenic and therapeutic agents. Cancer Res, Vol. 50, No. 19, (Oct 1) pp. 6119-6129.

Pelloski, C. E., et al. (2007). Epidermal growth factor receptor variant III status defines clinically distinct subtypes of glioblastoma. J Clin Oncol, Vol. 25, No. 16, (Jun 1) pp. 2288-2294. 
Perry, J. R., et al. (2010). Phase II trial of continuous dose-intense temozolomide in recurrent malignant glioma: RESCUE study. J Clin Oncol, Vol. 28, No. 12, (Apr 20) pp. 20512057.

Perry, J. R., et al. (2008). Temozolomide rechallenge in recurrent malignant glioma by using a continuous temozolomide schedule: the "rescue" approach. Cancer, Vol. 113, No. 8, (Oct 15) pp. 2152-2157.

Phillips, H. S., et al. (2006). Molecular subclasses of high-grade glioma predict prognosis, delineate a pattern of disease progression, and resemble stages in neurogenesis. Cancer Cell, Vol. 9, No. 3, (Mar) pp. 157-173.

Pierson, J., et al. (2008). Regulation of cyclin dependent kinase 6 by microRNA 124 in medulloblastoma. J Neurooncol, Vol. 90, No. 1, (Oct) pp. 1-7.

Pillai, R. S., et al. (2005). Inhibition of translational initiation by Let-7 MicroRNA in human cells. Science, Vol. 309, No. 5740, (Sep 2) pp. 1573-1576.

Prados, M., et al. (2011). Response as a predictor of survival in patients with recurrent glioblastoma treated with bevacizumab. Neuro Oncol, Vol. 13, No. 1, (Jan) pp. 143151.

Prados, M. D., et al. (2009). Phase II study of erlotinib plus temozolomide during and after radiation therapy in patients with newly diagnosed glioblastoma multiforme or gliosarcoma. J Clin Oncol, Vol. 27, No. 4, (Feb 1) pp. 579-584.

Prasad, G., et al. (2011). Inhibition of PI3K/mTOR pathways in glioblastoma and implications for combination therapy with temozolomide. Neuro Oncol, Vol. 13, No. 4, (Apr) pp. 384-392.

Preusser, M., et al. (2008). Anti-O6-methylguanine-methyltransferase (MGMT) immunohistochemistry in glioblastoma multiforme: observer variability and lack of association with patient survival impede its use as clinical biomarker. Brain Pathol, Vol. 18, No. 4, (Oct) pp. 520-532.

Puzanov, I., et al. (2011). Biological challenges of BRAF inhibitor therapy. Mol Oncol, Vol. 5, No. 2, (Apr) pp. 116-123.

Quinn, J. A., et al. (2005). Phase I trial of temozolomide plus O6-benzylguanine for patients with recurrent or progressive malignant glioma. J Clin Oncol, Vol. 23, No. 28, (Oct 1 ) pp. 7178-7187.

Quinn, J. A., et al. (2002). Phase II trial of carmustine plus $\mathrm{O}(6)$-benzylguanine for patients with nitrosourea-resistant recurrent or progressive malignant glioma. J Clin Oncol, Vol. 20, No. 9, (May 1) pp. 2277-2283.

Ramirez, C., et al. (2010). Loss of 1p, 19q, and 10q heterozygosity prospectively predicts prognosis of oligodendroglial tumors--towards individualized tumor treatment? Neuro-oncology, Vol. 12, No. 5, pp. 490-499.

Reardon, D. A., et al. (2010). Phase 2 trial of erlotinib plus sirolimus in adults with recurrent glioblastoma. J Neurooncol, Vol. 96, No. 2, (Jan) pp. 219-230.

Reardon, D. A., et al. (2011). Cilengitide: an RGD pentapeptide alphanubeta3 and alphanubeta5 integrin inhibitor in development for glioblastoma and other malignancies. Future Oncol, Vol. 7, No. 3, (Mar) pp. 339-354.

Reitman, Z. J., et al. (2011). Profiling the effects of isocitrate dehydrogenase 1 and 2 mutations on the cellular metabolome. Proc Natl Acad Sci U S A, Vol. 108, No. 8, (Feb 22) pp. 3270-3275.

Reitman, Z. J., et al. (2010). IDH1 and IDH2: not your typical oncogenes. Cancer Cell, Vol. 17, No. 3, (Mar 16) pp. 215-216. 
Reitman, Z. J. and H. Yan (2010). Isocitrate dehydrogenase 1 and 2 mutations in cancer: alterations at a crossroads of cellular metabolism. J Natl Cancer Inst, Vol. 102, No. 13, (Jul 7) pp. 932-941.

Ruano, Y., et al. (2009). Worse outcome in primary glioblastoma multiforme with concurrent epidermal growth factor receptor and p53 alteration. Am J Clin Pathol, Vol. 131, No. 2, (Feb) pp. 257-263.

Saidi, A., et al. (2009). Combined targeting of interleukin-6 and vascular endothelial growth factor potently inhibits glioma growth and invasiveness. Int J Cancer, Vol. 125, No. 5, (Sep 1) pp. 1054-1064.

Salter, K. H., et al. (2008). An integrated approach to the prediction of chemotherapeutic response in patients with breast cancer. PLoS One, Vol. 3, No. 4, pp. e1908.

Sampson, J. H., et al. (2011). Greater chemotherapy-induced lymphopenia enhances tumorspecific immune responses that eliminate EGFRvIII-expressing tumor cells in patients with glioblastoma. Neuro Oncol, Vol. 13, No. 3, (Mar) pp. 324-333.

Sasayama, T., et al. (2009). MicroRNA-10b is overexpressed in malignant glioma and associated with tumor invasive factors, uPAR and RhoC. Int J Cancer, Vol. 125, No. 6, (Sep 15) pp. 1407-1413.

Sawlani, R. N., et al. (2011). Glioblastoma: a method for predicting response to antiangiogenic chemotherapy by using MR perfusion imaging--pilot study. Radiology, Vol. 255, No. 2, (May) pp. 622-628.

Server, A., et al. (2011). Diagnostic examination performance by using microvascular leakage, cerebral blood volume, and blood flow derived from 3-T dynamic susceptibility-weighted contrast-enhanced perfusion MR imaging in the differentiation of glioblastoma multiforme and brain metastasis. Neuroradiology, Vol. 53, No. 5, (May) pp. 319-330.

Settle, S. H. and E. P. Sulman (2011). Tumor profiling: development of prognostic and predictive factors to guide brain tumor treatment. Curr Oncol Rep, Vol. 13, No. 1, (Feb) pp. 26-36.

Shi, L., et al. (2008). hsa-mir-181a and hsa-mir-181b function as tumor suppressors in human glioma cells. Brain Res, Vol. 1236, No., (Oct 21) pp. 185-193.

Shinojima, N., et al. (2003). Prognostic value of epidermal growth factor receptor in patients with glioblastoma multiforme. Cancer Res, Vol. 63, No. 20, (Oct 15) pp. 6962-6970.

Shiraishi, S., et al. (2002). Influence of p53 mutations on prognosis of patients with glioblastoma. Cancer, Vol. 95, No. 2, (Jul 15) pp. 249-257.

Silber, J., et al. (2008). miR-124 and miR-137 inhibit proliferation of glioblastoma multiforme cells and induce differentiation of brain tumor stem cells. BMC Med, Vol. 6, No., pp. 14.

Silber, J. R., et al. (1998). O6-methylguanine-DNA methyltransferase activity in adult gliomas: relation to patient and tumor characteristics. Cancer Res, Vol. 58, No. 5, (Mar 1) pp. 1068-1073.

Sorensen, A. G., et al. (2009). A "vascular normalization index" as potential mechanistic biomarker to predict survival after a single dose of cediranib in recurrent glioblastoma patients. Cancer Res, Vol. 69, No. 13, (Jul 1) pp. 5296-5300.

Sreekanthreddy, P., et al. (2010). Identification of potential serum biomarkers of glioblastoma: serum osteopontin levels correlate with poor prognosis. Cancer Epidemiol Biomarkers Prev, Vol. 19, No. 6, (Jun) pp. 1409-1422. 
Stingl, J. C., et al. (2010). Impact of CYP2D6*4 genotype on progression free survival in tamoxifen breast cancer treatment. Curr Med Res Opin, Vol. 26, No. 11, (Nov) pp. 2535-2542.

Stupp, R., et al. (2009). Effects of radiotherapy with concomitant and adjuvant temozolomide versus radiotherapy alone on survival in glioblastoma in a randomised phase III study: 5-year analysis of the EORTC-NCIC trial. Lancet Oncol, Vol. 10, No. 5, (May) pp. 459-466.

Stupp, R., et al. (2010). Phase I/IIa study of cilengitide and temozolomide with concomitant radiotherapy followed by cilengitide and temozolomide maintenance therapy in patients with newly diagnosed glioblastoma. J Clin Oncol, Vol. 28, No. 16, (Jun 1) pp. 2712-2718.

Stupp, R., et al. (2005). Radiotherapy plus concomitant and adjuvant temozolomide for glioblastoma. N Engl J Med, Vol. 352, No. 10, (Mar 10) pp. 987-996.

Tews, B., et al. (2006). Identification of novel oligodendroglioma-associated candidate tumor suppressor genes in 1p36 and 19q13 using microarray-based expression profiling. Int J Cancer, Vol. 119, No. 4, (Aug 15) pp. 792-800.

Tews, B., et al. (2007). Hypermethylation and transcriptional downregulation of the CITED4 gene at $1 \mathrm{p} 34.2$ in oligodendroglial tumours with allelic losses on $1 \mathrm{p}$ and $19 \mathrm{q}$. Oncogene, Vol. 26, No. 34, (Jul 26) pp. 5010-5016.

Thiessen, B., et al. (2010). A phase I/II trial of GW572016 (lapatinib) in recurrent glioblastoma multiforme: clinical outcomes, pharmacokinetics and molecular correlation. Cancer Chemother Pharmacol, Vol. 65, No. 2, (Jan) pp. 353-361.

Tolcher, A. W., et al. (2003). Marked inactivation of O-6-alkylguanine-DNA alkyltransferase activity with protracted temozolomide schedules. British Journal Of Cancer, Vol. 88, No. 7, (Apr 7) pp. 1004-1011.

Tsien, C., et al. (2011). Parametric response map as an imaging biomarker to distinguish progression from pseudoprogression in high-grade glioma. J Clin Oncol, Vol. 28, No. 13, (May 1) pp. 2293-2299.

Uhm, J. H., et al. (2010). Phase II Evaluation of Gefitinib in Patients with Newly Diagnosed Grade 4 Astrocytoma: Mayo/North Central Cancer Treatment Group Study N0074. Int J Radiat Oncol Biol Phys, Vol., No., (May 24).

van den Bent, M. J., et al. (2009). Randomized phase II trial of erlotinib versus temozolomide or carmustine in recurrent glioblastoma: EORTC brain tumor group study 26034. J Clin Oncol, Vol. 27, No. 8, (Mar 10) pp. 1268-1274.

van den Bent, M. J., et al. (2006). Adjuvant procarbazine, lomustine, and vincristine improves progression-free survival but not overall survival in newly diagnosed anaplastic oligodendrogliomas and oligoastrocytomas: a randomized European Organisation for Research and Treatment of Cancer phase III trial. J Clin Oncol, Vol. 24, No. 18, (Jun 20) pp. 2715-2722.

Verhaak, R. G., et al. (2010). Integrated genomic analysis identifies clinically relevant subtypes of glioblastoma characterized by abnormalities in PDGFRA, IDH1, EGFR, and NF1. Cancer Cell, Vol. 17, No. 1, (Jan 19) pp. 98-110.

Verhaak, R. G. and P. J. Valk (2010). Genes predictive of outcome and novel molecular classification schemes in adult acute myeloid leukemia. Cancer Treat Res, Vol. 145, No., pp. 67-83.

Vredenburgh, J. J., et al. (2007). Bevacizumab plus irinotecan in recurrent glioblastoma multiforme. J Clin Oncol, Vol. 25, No. 30, (Oct 20) pp. 4722-4729. 
Vredenburgh, J. J., et al. (2011). The Addition of Bevacizumab to Standard Radiation Therapy and Temozolomide Followed by Bevacizumab, Temozolomide and Irinotecan for Newly Diagnosed Glioblastoma. Clin Cancer Res, Vol., No., (Apr 29).

Walker, C., et al. (2006). Clinical use of genotype to predict chemosensitivity in oligodendroglial tumors. Neurology, Vol. 66, No. 11, (Jun 13) pp. 1661-1667.

Wang, R., et al. (2011). Glioblastoma stem-like cells give rise to tumour endothelium. Nature, Vol. 468, No. 7325, (Dec 9) pp. 829-833.

Wang, S. and E. N. Olson (2009). AngiomiRs--key regulators of angiogenesis. Curr Opin Genet Dev, Vol. 19, No. 3, (Jun) pp. 205-211.

Webster, R. J., et al. (2009). Regulation of epidermal growth factor receptor signaling in human cancer cells by microRNA-7. J Biol Chem, Vol. 284, No. 9, (Feb 27) pp. 5731-5741.

Wen, P. Y., et al. (2010). Updated response assessment criteria for high-grade gliomas: response assessment in neuro-oncology working group. J Clin Oncol, Vol. 28, No. 11, (Apr 10) pp. 1963-1972.

Whittaker, S., et al. (2010). Gatekeeper mutations mediate resistance to BRAF-targeted therapies. Sci Transl Med, Vol. 2, No. 35, (Jun 9) pp. 35ra41.

Wick, A., et al. (2007). Efficacy and tolerability of temozolomide in an alternating weekly regimen in patients with recurrent glioma. J Clin Oncol, Vol. 25, No. 22, (Aug 1) pp. 3357-3361.

Wick, W., et al. (2009). NOA-04 randomized phase III trial of sequential radiochemotherapy of anaplastic glioma with procarbazine, lomustine, and vincristine or temozolomide. J Clin Oncol, Vol. 27, No. 35, (Dec 10) pp. 5874-5880.

Wick, W., et al. (2010). Phase III study of enzastaurin compared with lomustine in the treatment of recurrent intracranial glioblastoma. J Clin Oncol, Vol. 28, No. 7, (Mar 1) pp. 1168-1174.

Wick, W., et al. (2011). Phase III study of enzastaurin compared with lomustine in the treatment of recurrent intracranial glioblastoma. J Clin Oncol, Vol. 28, No. 7, (Mar 1) pp. 1168-1174.

Wick, W., et al. (2004). One week on/one week off regimen of temozolomide for recurrent glioblastoma: A phase III study. Journal Of Clinical Oncology, Vol. 22, No. 14, (Jul 15) pp. 116S-116S.

Wick, W. and M. Weller (2005). How lymphotoxic is dose-intensified temozolomide? The glioblastoma experience. Journal Of Clinical Oncology, Vol. 23, No. 18, (Jun 20) pp. 4235-4236.

Wu, L. e. a. (2006). MicroRNA direct rapid demethylation of mRNA. PNAS, Vol. 103, No., pp. 4034-4039.

$\mathrm{Xu}, \mathrm{W}$. , et al. (2011). Oncometabolite 2-hydroxyglutarate is a competitive inhibitor of alphaketoglutarate-dependent dioxygenases. Cancer Cell, Vol. 19, No. 1, (Jan 18) pp. 17-30.

Yan, H., et al. (2009). IDH1 and IDH2 mutations in gliomas. N Engl J Med, Vol. 360, No. 8, (Feb 19) pp. 765-773.

Yekta, S. e. a. (2004). MicroRNA-directed cleavage of HOXB8 mRNA. Science, Vol. 304, No., pp. 594-596.

Zhang, Y., et al. (2009). MicroRNA-128 inhibits glioma cells proliferation by targeting transcription factor E2F3a. J Mol Med, Vol. 87, No. 1, (Jan) pp. 43-51. 


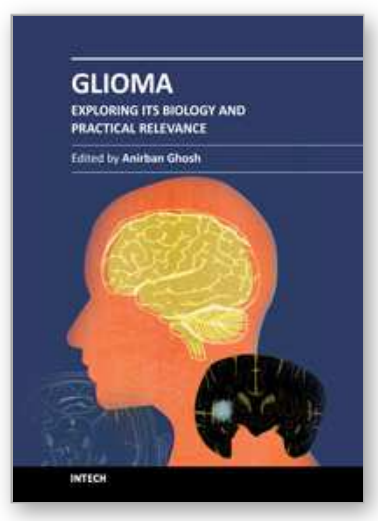

\author{
Glioma - Exploring Its Biology and Practical Relevance \\ Edited by Dr. Anirban Ghosh
}

ISBN 978-953-307-379-8

Hard cover, 486 pages

Publisher InTech

Published online 02, November, 2011

Published in print edition November, 2011

The tittle 'Glioma - Exploring Its Biology and Practical Relevance' is indicative of its content. This volume contains 21 chapters basically intended to explore glioma biology and discussing the experimental model systems for the purpose. It is hoped that the present volume will provide supportive and relevant awareness and understanding on the fundamental advances of the subject to the professionals from any sphere interested about glioma.

\title{
How to reference
}

In order to correctly reference this scholarly work, feel free to copy and paste the following:

Kerrie L. McDonald (2011). Biomarker Discovery, Validation and Clinical Application for Patients Diagnosed with Glioma, Glioma - Exploring Its Biology and Practical Relevance, Dr. Anirban Ghosh (Ed.), ISBN: 978-953307-379-8, InTech, Available from: http://www.intechopen.com/books/glioma-exploring-its-biology-andpractical-relevance/biomarker-discovery-validation-and-clinical-application-for-patients-diagnosed-with-glioma

\section{INTECH}

open science | open minds

\section{InTech Europe}

University Campus STeP Ri

Slavka Krautzeka 83/A

51000 Rijeka, Croatia

Phone: +385 (51) 770447

Fax: +385 (51) 686166

www.intechopen.com

\section{InTech China}

Unit 405, Office Block, Hotel Equatorial Shanghai

No.65, Yan An Road (West), Shanghai, 200040, China

中国上海市延安西路65号上海国际贵都大饭店办公楼 405 单元

Phone: +86-21-62489820

Fax: +86-21-62489821 
(C) 2011 The Author(s). Licensee IntechOpen. This is an open access article distributed under the terms of the Creative Commons Attribution 3.0 License, which permits unrestricted use, distribution, and reproduction in any medium, provided the original work is properly cited. 